\title{
Structure and nanomechanics of dry and hydrated intermediate filament films and fibers produced from hagfish slime fibers
}

\author{
L J Böni ${ }^{1 \neq}$, A Sanchez-Ferrer², M Widmer ${ }^{1}$, M D Biviano³ ${ }^{3}$ R Mezzenga² ${ }^{2}$ E J Windhab ${ }^{1}$, R R \\ Dagastine $^{3}$, and P Fischer ${ }^{1}$
}

${ }^{1}$ Food Process Engineering, Department of Health Science and Technology, ETH Zürich, 8092 Zürich, Switzerland

${ }^{2}$ Food and Soft Materials, Department of Health Science and Technology, ETH Zürich, 8092 Zürich, Switzerland

${ }^{3}$ Department of Chemical and Biomolecular Engineering, University of Melbourne, Melbourne, Australia

‡Corresponding author email: lukas.boeni@hest.ethz.ch

Key words: intermediate filaments, hagfish slime, swelling, nanoindentation, viscoelasticity

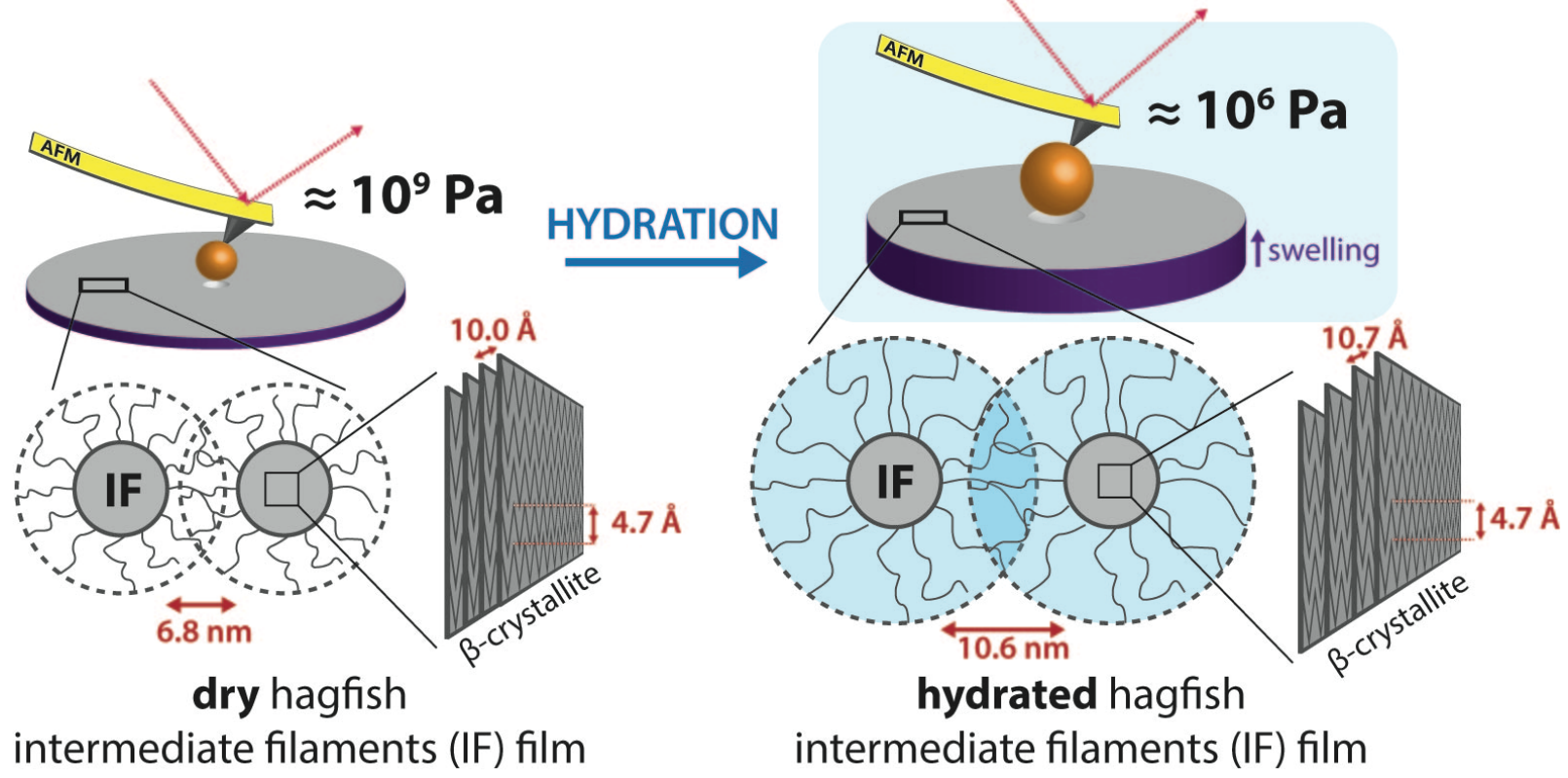

TOC Figure: Intermediate filament (IF) films produced from hagfish fiber protein readily hydrate and change from a stiff $\left(10^{9} \mathrm{~Pa}\right)$ to a soft $\left(10^{6} \mathrm{~Pa}\right)$ viscoelastic material.

\begin{abstract}
Intermediate filaments (IFs) are known for their extensibility, flexibility, toughness, and their ability to hydrate. Using keratin-like IFs obtained from slime fibers from the invertebrate Atlantic hagfish (M. glutinosa), films were produced by drop-casting and coagulation on the surface of a $\mathrm{MgCl}_{2}$ buffer. Drop-casting produced self-supporting, smooth, and dense films rich in $\beta$-sheets $(61 \%)$ whereas coagulation formed thin, porous films with a nano-rough surface and a lower $\beta$ sheet content $(51 \%)$. The films hydrated and swelled immediately when immersed in water and did not dissolve. X-ray diffraction showed that the $\beta$-crystallites remained stable upon hydration, that swelling presumably happens in the amorphous $\mathrm{C}$-terminal tail-domains of the IFs, and that high salt conditions caused a denser network mesh-size, suggesting polyelectrolyte behavior.
\end{abstract}


Hydration resulted in a roughly thousandfold decrease in apparent Young's modulus from $10^{9}$ to $10^{6} \mathrm{~Pa}$ as revealed by AFM nanoindentation. Nanoindentation-based power-law rheology (PLR) and stress-relaxation measurements indicated viscoelasticity and a soft-solid hydrogel character for hydrated films, where roughly $80 \%$ of energy is elastically stored and $20 \%$ is dissipated. By pulling coagulation films from the buffer interface, macroscopic fibers with highly aligned IF $\beta$ crystals similar to natural hagfish fibers were produced. We propose that viscoelasticity and strong hydrogen bonding interactions with the buffer interface are crucial for the production of such long biomimetic fibers with aligned $\beta$-sheets. This study demonstrates that hagfish fiber IFs can be reconstituted into functional biomimetic materials that are stiff when dry and retain the ability to hydrate to become soft and viscoelastic when in water.

\section{Introduction}

Intermediate filaments (IFs) are a major structural element of animal cells, playing an important role in cell mechanics by acting as stress absorbers, imparting integrity, and by mediating mechanotransduction processes ${ }^{1-6}$. IF have a diameter of approximately $10 \mathrm{~nm}$ and share a common architecture characterized by of a central $\alpha$-helical coiled-coil 'rod' domain, flanked by a largely amorphous N-terminal 'head' and C-terminal 'tail' domain of variable length and sequence $^{7}$. Given their open molecular architecture and their unique assembly plan ${ }^{4}$, IFs possess unique mechanical properties ${ }^{8-10}$ by combining extreme extensibility ${ }^{11-13}$, flexibility ${ }^{14}$, and toughness, making them much more flexible and resistant to large deformations than other cytoskeletal polymers such as F-actin and microtubules ${ }^{13,15,16}$.

A rich source of keratin-like IFs can be found in hagfish slime threads ${ }^{17-21}$, commonly referred to as hagfish fibers or hagfish threads. The invertebrate hagfish are famous for their ability to produce vast amounts of defensive slime when attacked or stressed ${ }^{22,23}$. In contrast to other mucous slimes that are made primarily of mucin-like molecules, hagfish slime also contains long threads, which make it a cohesive, strong, and soft-elastic biomaterial ${ }^{24-27}$. Hagfish threads consist of axially aligned IFs, which condense to a solid 1 - $3 \mu \mathrm{m}$ diameter fiber that can be up to $15 \mathrm{~cm}$ long ${ }^{17,25,28,29}$. The threads show a typical "alpha-pattern" in X-ray measurements with a meridional reflection at $5.15 \AA$ and an equatorial reflection at $9.8 \AA$, indicating the presence of helical structures. When large strains act on the threads, the a-helical domains in the coiledcoils begin to extend into $\beta$-strands, resulting in an irreversible $\alpha \rightarrow \beta$ conformational transition of the IFs ${ }^{24}$. Hagfish IFs comprise three thread keratin (TK) proteins $(\alpha, \beta \text {, and } \gamma)^{20}$, whereby $\beta$ is likely a post-translationally modified version of $\gamma^{18}$. They are classified as IFs because they contain a central rod domain with heptad repeat motifs of apolar residues, rod subdomains shared with other IF proteins, a 'stutter' of the heptad repeat in subdomain $2 \mathrm{~B}$, conserved sequences at each end of the rod domain, and non-helical $\mathrm{N}$ - and C-terminal domains that connect to the central rod domain ${ }^{19,21,30}$. Hagfish IFs were termed 'keratin-like' due to features in the head and tail domains that are similar to keratin ${ }^{19,21}$. TKa was found to be a type II keratin homolog and TKY possesses features of type I keratins, but also contains structural similarities to type III IFs, which include desmin and vimentin ${ }^{30,31}$. Furthermore, TKa and TKY were found to self-assemble into hetero-dimeric IF like nanofibrils in vitro ${ }^{20,32,33}$. Hagfish fibers still comprise the only known example of extracellularly secreted IFs. As in all other known cases, IFs occur 
strictly intracellularly ${ }^{29,34}$. Mechanical properties similar to spider silk ${ }^{24}$, as well as the high protein concentration (supposed to approach $1000 \mathrm{mg} / \mathrm{mL}$ in hydrated threads ${ }^{35}$ ), and the ability to isolate relatively large (several grams) quantities ${ }^{17}$ ) make hagfish fibers an intriguing natural source of invertebrate IFs.

Recently, macroscopic fibers were produced from naturally sourced ${ }^{36}$ and bacterially expressed $^{32,33}$ hagfish IFs from the Pacific hagfish (E. stoutii). Whereas these studies focused on the mechanical performance of the dry reconstituted fibers, little is known about the ability of reconstituted IF materials to hydrate and the associated changes in mechanical properties. Hydration differs strongly for hard and soft $\alpha$-keratins and is a key property of IFs. In hard $\alpha$ keratin such as hair and nails, the approx. $10 \mathrm{~nm}$ IF fibrils are embedded in an isotropic, highsulphur matrix ${ }^{37}$, keeping them in a largely hydration-free state ${ }_{2}^{35}$ and thus forming strong composite materials ${ }^{38,39}$. In soft $\alpha$-keratin as found in the stratum corneum (soft outer layer of skin), hydrated IFs are - apart from their mechanical role - crucial for water conservation ${ }^{40}$. In contrast to F-actin and microtubules, which exist in a dynamic equilibrium with soluble pools of monomers within cells, IFs are much less soluble ${ }_{20,41}$ and therefore constitute a good source of functional protein for biomaterials with potential applications in liquid environments.

In this study we investigated the mechanical properties and protein secondary structure of IF films produced from hagfish fibers in the dry and hydrated state. Using naturally sourced IFs from thread skeins of the Atlantic hagfish (M. glutinosa), two different methods were used to produce films: i) drop casting, and ii) coagulation on a magnesium chloride bath as first described by Negishi et al. ${ }^{36}$. The films were compared for their secondary structure assessed by Fourier transform infrared spectroscopy (FTIR) and X-ray diffraction (XRD), as well as their surface morphology using AFM imaging. Films were then hydrated and structural changes (swelling height, network mesh-size) as well as viscoelasticity assessed by nanoindentation were monitored and compared to the dry state. In a last part, biomimetic fibers with aligned $\beta$ sheets similar to natural hagfish fibers were produced and implications of IF viscoelasticity on the production of such bioinspired materials are discussed. Novel materials made from hagfish IFs that show high swellability without dissolution could potentially be used in scaffolds for ocular surface reconstruction as shown for keratin ${ }^{42}$, in smart textiles which show shapemorphing in response to external humidity ${ }^{43,44}$, as edible coatings for food preservation in high moisture environments as similarly reported for spider silk ${ }^{45}$, or as non-lethal and non-kinetic naval defense to protect ships from ballistic projectiles as investigated by the US Navy ${ }^{46}$. Our observations provide valuable insights into the mechanics of condensed IF systems and their response to hydration and will support the design of such novel bioinspired IF materials.

\section{Results and Discussion}

\section{Isolation of intermediate filament protein from hagfish skeins}

Intermediate filament (IF) proteins were obtained from hagfish thread skeins - coiled up hagfish fibers - from the Atlantic hagfish ( $M$. glutinosa). The skeins were separated from the mucin vesicles via filtration, washed with Milli-Q water, dialysed and freeze-dried. The isolated dry skeins (Figure 1a) and mucin (Figure 1b) were checked for purity using SDS-PAGE gel electrophoresis (Figure S1a,b,c) and FTIR (Figure 1c). In SDS-PAGE, the skeins showed a major double band with molecular weights of $62 \mathrm{kDa}$ and $67 \mathrm{kDa}$, which agrees well with the 
findings from Koch et al. ${ }^{19,21}$ who reported a molecular weight of $66.6 \mathrm{KDa}$ for the $\alpha$-subunit and 62.7 for the $y$-subunit of the IF heterodimer for the Pacific hagfish ( $E$. stoutii). Additionally, two minor bands are visible at about $53 \mathrm{kDa}$ and $58 \mathrm{kDa}$. They could originate from small amounts of microtubules ( $\alpha$ - and $\beta$-tubulin of approx. $50 \mathrm{kDa}^{47}$ ), which occur within skeins that are not fully mature ${ }^{17,48}$.
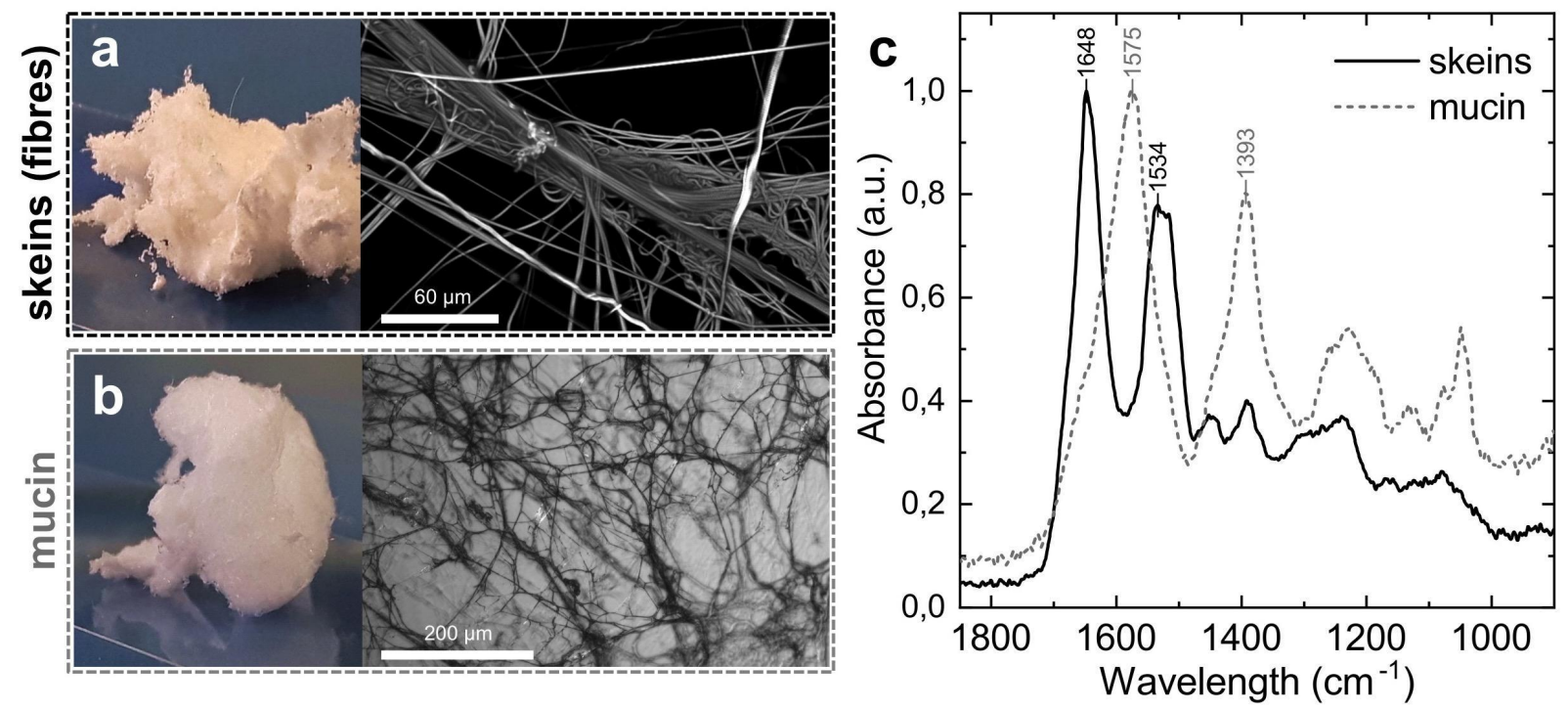

Figure 1: Separation hagfish fiber intermediate filament (IF) protein from hagfish mucin. (a) Photograph of freeze-dried hagfish skeins on a microscopy slide (left), and confocal scanning laser microscopy (CLSM) of hagfish slime fibers (uncoiled skeins) in water (right). (b) Photograph of freeze-dried hagfish mucin on a microscopy slide (left) and corresponding CLSM image (right). (c) ATR-FTIR spectra of freeze-dried skeins and mucin (the spectra were normalized to their highest peak for comparison).

Mucin showed a major band at $89 \mathrm{kDa}$ and a minor band at $37 \mathrm{kDa}$ and one component that did not enter the gel, as similarly observed for mucin isolated from the Pacific hagfish ${ }^{20,49}$. The major band at $89 \mathrm{kDa}$ is in good agreement with findings of Spitzer et al. ${ }^{20}$ but differs from findings of Salo et al. ${ }^{49}$. Both components - skeins and mucins - showed distinct FTIR spectra (Figure 1c), supporting their successful separation. The skein fraction showed a spectrum characteristic for proteins with an amide I peak around $\sim 1648 \mathrm{~cm}^{-1}$ and amide II peak around $\sim 1534 \mathrm{~cm}^{-1}$. In contrast, the mucin fraction had a clearly different spectrum with a major peak around $1575 \mathrm{~cm}^{-1}$ and $1393 \mathrm{~cm}^{-1}$. Hagfish mucin is considered a large, highly sulfated glycoprotein ${ }^{49,50}$. The peak around $1575 \mathrm{~cm}^{-1}$ shows shoulders at the amide I and II position, indicating the presence of protein but shifted, probably because of sugars bound to the protein backbone. The prominent peak around $1393 \mathrm{~cm}^{-1}$ could result from protein side-chain COO'. The peak around $1230 \mathrm{~cm}^{-1}$ could originate from the antisymmetric $\mathrm{S}=\mathrm{O}$ stretch vibration from sulfates, which is typical for highly sulfated carbohydrates ${ }^{51}$ and was also observed for sputum $^{52}$. The peak around $1050 \mathrm{~cm}^{-1}$ is an indication for carbohydrates such as $\mathrm{N}$ acetylglucosamine, $\mathrm{N}$-acetylgalactosamine, and sialic acid bound to mucins ${ }^{52}$. These peaks were also observed for other mucins such as porcine gastric mucin, bovine submaxillary 
mucin $^{53-55}$ and invertebrate mucins ${ }_{26,57}$ and agree well with the reported sugars in hagfish $\operatorname{mucin}^{49,50}$.

\section{Hagfish IF films are rich in $\beta$-sheets}

Separated and freeze-dried skeins (IF protein) were solubilized in formic acid and two different approaches were used to produce films: i) drop-casting, and ii) coagulation on the surface of an ice-cold buffer bath containing $\mathrm{MgCl}_{2}$ (Figure 2a). Both films were partly transparent. Whereas drop-casting produced thick, self-supporting, and highly birefringent films (Figure 2b,d), coagulation resulted in thin, brittle, and weakly birefringent films (Figure $2 c, e$ ). The stronger birefringence of the drop-cast film likely originates in the increased thickness and could additionally be favoured by a higher $\beta$-sheet content. FTIR peak deconvolution revealed that both films mainly consisted of $\beta$-sheets $\left(\sim 1620 \mathrm{~cm}^{-1}\right)$, random coils $\left(\sim 1650 \mathrm{~cm}^{-1}\right)$, and a smaller amount of $\beta$-turns $\left(\sim 1680 \mathrm{~cm}^{-1}\right)$ (Figure $2 \mathrm{f}, \mathrm{g}$ ). The drop-cast film contained higher amounts of beta-population (61\%) compared to the coagulation film (49\%), and lower amounts of random coils (39\%) compared to the coagulation film (49\%). The observed 'cross' in birefringence (Figure $2 b$ ) suggests an orientation of IF $\beta$-crystallites in the middle of the film where the $\beta$ sheets in the IF $\beta$-crystallites are perpendicular to the incident light, and a radial planar orientation in the outer rim where $\beta$-sheets in the IF $\beta$-crystallites are not. In order to confirm this idea, the film was tilted between the cross-polarizers and the middle part also became birefringent. Additionally, a homogeneous orientation of the IF $\beta$-crystallites was observed when the film was cast on glass (Figure S2). The different $\beta$-crystallite orientations between films cast on teflon and films cast on glass probably stem from a combination of dissimilar surface tension effects during drying and the different wetting of teflon (hydrophobic) and glass (hydrophilic).
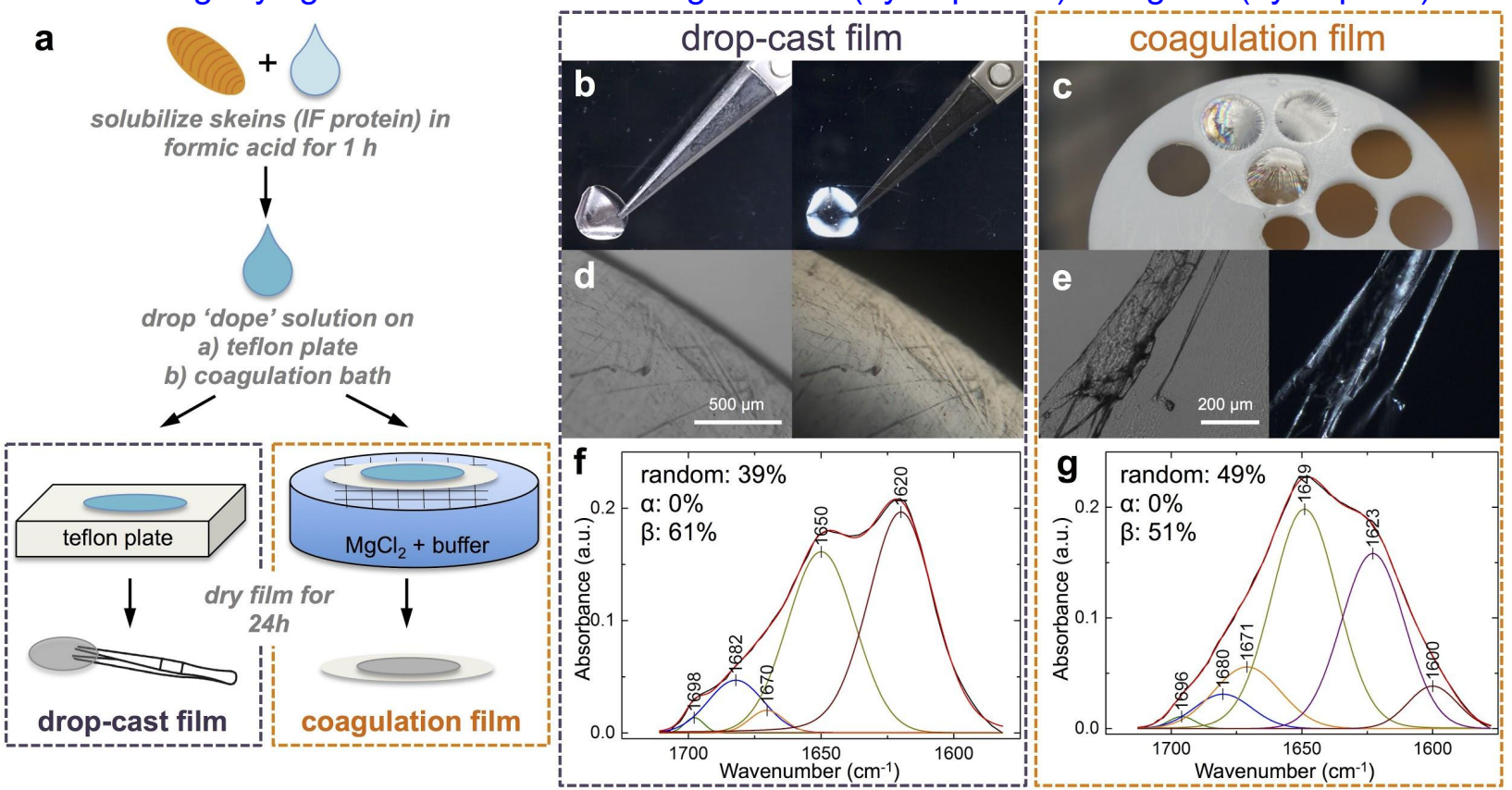

Figure 2: Production, morphology, and protein secondary structure of films produced from hagfish fibers. (a) Film production from solubilized hagfish fibers. Hagfish IF protein is solubilized in formic acid and then either coagulated on a buffer containing $\mathrm{MgCl}_{2}$ or drop-cast 
on a teflon plate. From the buffer the film is removed with the help of a fine supportive metal mesh that is placed under a thin teflon plate with holes. Both films are air dried for $24 \mathrm{~h}$. Drop casting forms self-supporting films, which show strong birefringence (b) in contrast to the coagulation method, which forms thin films (c). Birefringence assessed with polarized light microscopy (d,e). For the thin coagulation film birefringence is only visible when several layers are on top of each other (scale bars are located on the films). FTIR peak deconvolution of a drop-cast (f) and coagulation film (g).

Whereas $\beta$-sheet rich structures were similarly found for fibers produced from natural ${ }^{36}$ and recombinant ${ }^{32}$ hagfish protein - where coagulation films were drawn from the water surface into macroscopic fibers - no $\alpha$-helices were observed in this study. Negishi et al. ${ }^{36}$ reported $\alpha$-helices identified by Raman spectroscopy. However, their deconvoluted spectra would alternatively allow to identify the peaks at $1654 \mathrm{~cm}^{-1}$ and $1655 \mathrm{~cm}^{-1}$ as random coils instead of a-helices given that peaks between 1647 and $1655 \mathrm{~cm}^{-1}$ are often assigned to random coils, and peaks between 1656 and $1662 \mathrm{~cm}^{-1}$ are often assigned to a-helices ${ }^{58}$ - and wide angle $\mathrm{x}$-ray scattering (WAXS) measurements did not show the presence of a-helices either. Based on circular dichroism measurements $\mathrm{Fu}$ et al. $^{32}$ suggested that a-helices (coiled-coils) are present in hagfish IFs when they are solubilized in formic acid but undergo an $\alpha$-helix to $\beta$-sheet ( $\alpha-\beta)$ transition at the water/air interface during picking up the film and drawing it into a fiber. Conformational changes of proteins at interfaces are a well-known phenomenon ${ }^{59}$. It is possible that the higher amount of $\beta$-sheets in the drop cast film compared to the coagulation films originates in its longer drying kinetics, leaving more time for conformational changes at the formic acid/air interface. However, given that stretching of the films was largely avoided during lifting the films from the water surface and that drop-cast films were not subjected to any stretching strains except surface tension effects during drying raises the question whether ahelices are at all present in materials produced by solubilizing hagfish fiber protein in formic acid. The absence of a-helices thus remains elusive so far but could be linked to the strongly chaotropic nature of formic acid, to the freeze-drying step in sample preparation ${ }^{60}$, or to the conformational changes at the air/water interface.

\section{Nano surface topology of IF films}

The height of coagulation films was typically in the range of $1 \mu \mathrm{m}$ and is limited by the spreading of the dope droplet at the buffer interface and the subsequent film formation. A film thickness of 2 - $4 \mu \mathrm{m}$ for drop cast films was targeted for AFM measurements (Figure 3a,b) but in contrast to coagulation films the height of drop-cast films can be tuned by protein concentration, cast volume, or by using a mold that can easily reach up to $50-100 \mu \mathrm{m}$. Both films appeared flat at the micron scale but showed distinct differences in surface morphology at the nanoscale (Figure 3c,d, Figure S3 a,b). The drop-cast film was denser and substantially smoother than the coagulation film and occasionally showed shallow and round dents that probably stem from surface tension stresses during air-drying. The coagulation film had a more nano-porous and nano-rough surface, which seems a result of the immediate solidification at the $\mathrm{MgCl}_{2}$ buffer surface. 

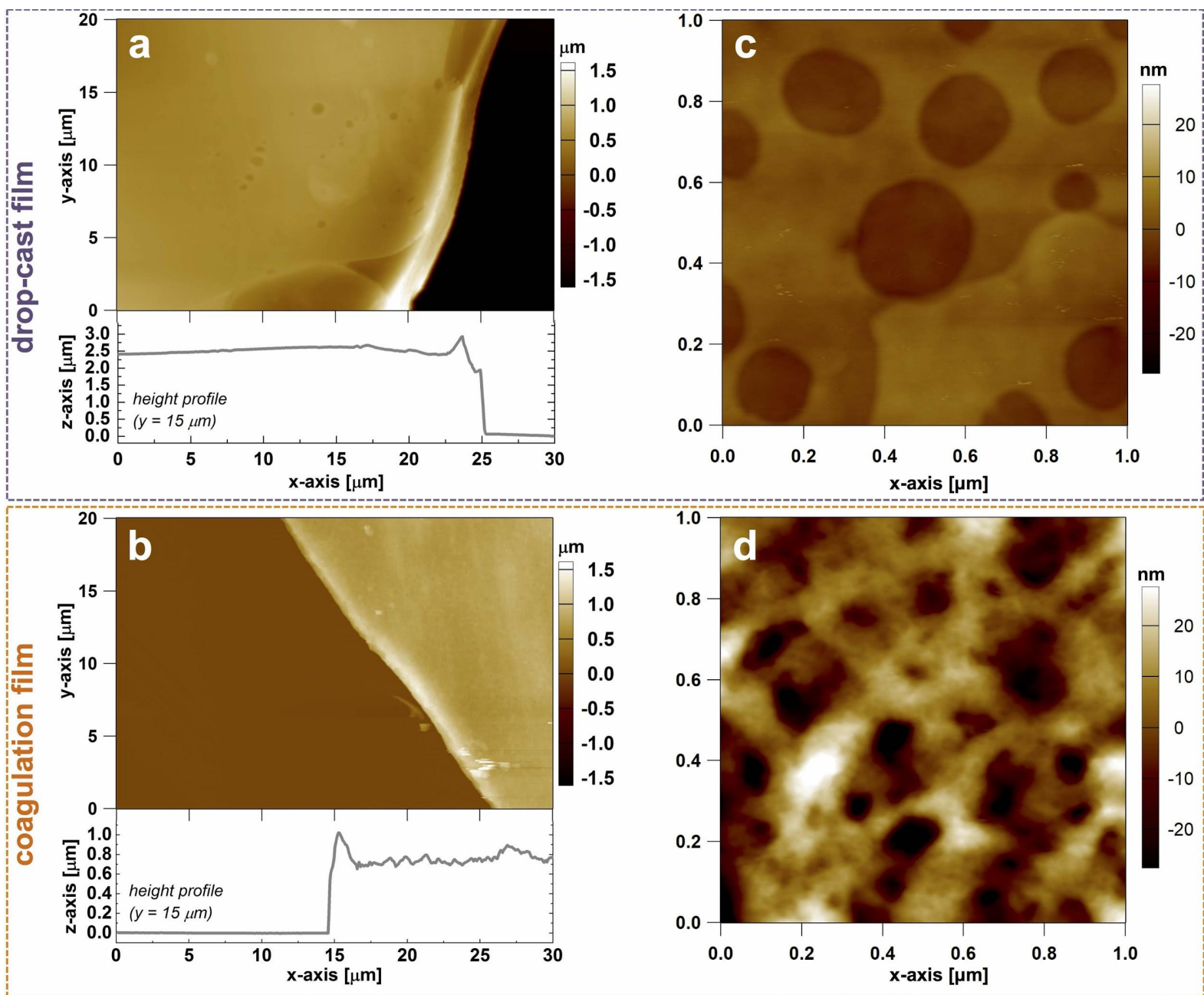

Figure 3: AFM height images showing the micro- and nano-topography of dry hagfish IF films scratched with a scalpel. Drop-cast (a) and coagulation film (b) show a smooth surface at the micro-scale. The height profile (z-data) at $y=15 \mu \mathrm{m}$ is shown below the images. At the nano-scale the drop-cast film (c) is considerably smoother than the coagulation film (d).

\section{Hagfish IF films readily hydrate and swell in water}

The reconstituted films readily hydrated and swelled when immersed in water, similar to native hagfish slime fibers ${ }^{24,35}$. Immersion in deionized water (Milli-Q), as well as in higher ionic strength solutions, did not dissolve the IF films - a hallmark feature of IFs ${ }^{61}$ - and the birefringence was conserved (Figure 4a). Hydration in 1\% simplified seawater (SSW; $5.45 \mathrm{mM}$ $\mathrm{NaCl}+0.1 \mathrm{mM} \mathrm{CaCl}_{2}$ ) resulted in immediate swelling of the films, whereby the drop-cast films increased $55 \pm 9 \%$ and coagulation films increased $73 \pm 13 \%$ in height (Figure $4 \mathrm{~b}$ ). In contrast to the coagulation film, hydration did not change the surface roughness of the drop-cast film (Figure 4c, Figure S3 c,d). Wide angle x-ray scattering (WAXS) measurements of dry films (Figure $4 \mathrm{~d}$ ) showed the presence of $\beta$-crystallites, indicated by the two peaks corresponding to $4.7 \AA$ (inter $\beta$-strand) and to $10.0 \AA$ (inter $\beta$-sheet) ${ }^{62}$ distances and thus supporting the FTIR findings. Upon hydration, the peak at $4.7 \AA$ remained unchanged, while the second peak at 10.0 
$\AA$ increased to $11.0 \AA$ when hydrated with Milli- $Q$ water, showing a lager inter $\beta$-sheet distance. These results demonstrate that the $\beta$-crystallites do not dissolve and supports the observation that birefringence does not vanish upon hydration. The main broad peak in the WAXS profile at ca. $\mathrm{q}=18 \mathrm{~nm}^{-1}$ for the swollen samples - indication of water molecules -, as well as the occurrence of a characteristic length $d=11.5 \mathrm{~nm}$ (Milli-Q) in small angle $x$-ray scattering (SAXS) measurements (Figure 4d) suggest that swelling mainly happens in the amorphous C-terminal tail domains of the IFs as already suggested for hagfish fibers ${ }^{24}$. The characteristic length likely represents an inter-IF distance and can be attributed to the average mesh-size of the gel system when films are hydrated. The mesh-size could be influenced by a bottle-brush configuration of the tail domains that are known to radially graft IFs (Figure 4e), whereby in average $16 \mathrm{IF}$ tails radiate from the filament backbone every $22 \mathrm{~nm}$, resulting in a distance of $6.6 \mathrm{~nm}$ between two neighbouring tails ${ }^{10,63,64}$. The above results suggest that hagfish IF films are semi-crystalline nanocomposites, consisting of $\beta$-crystallites (IF central part) and random coils (IF tails).
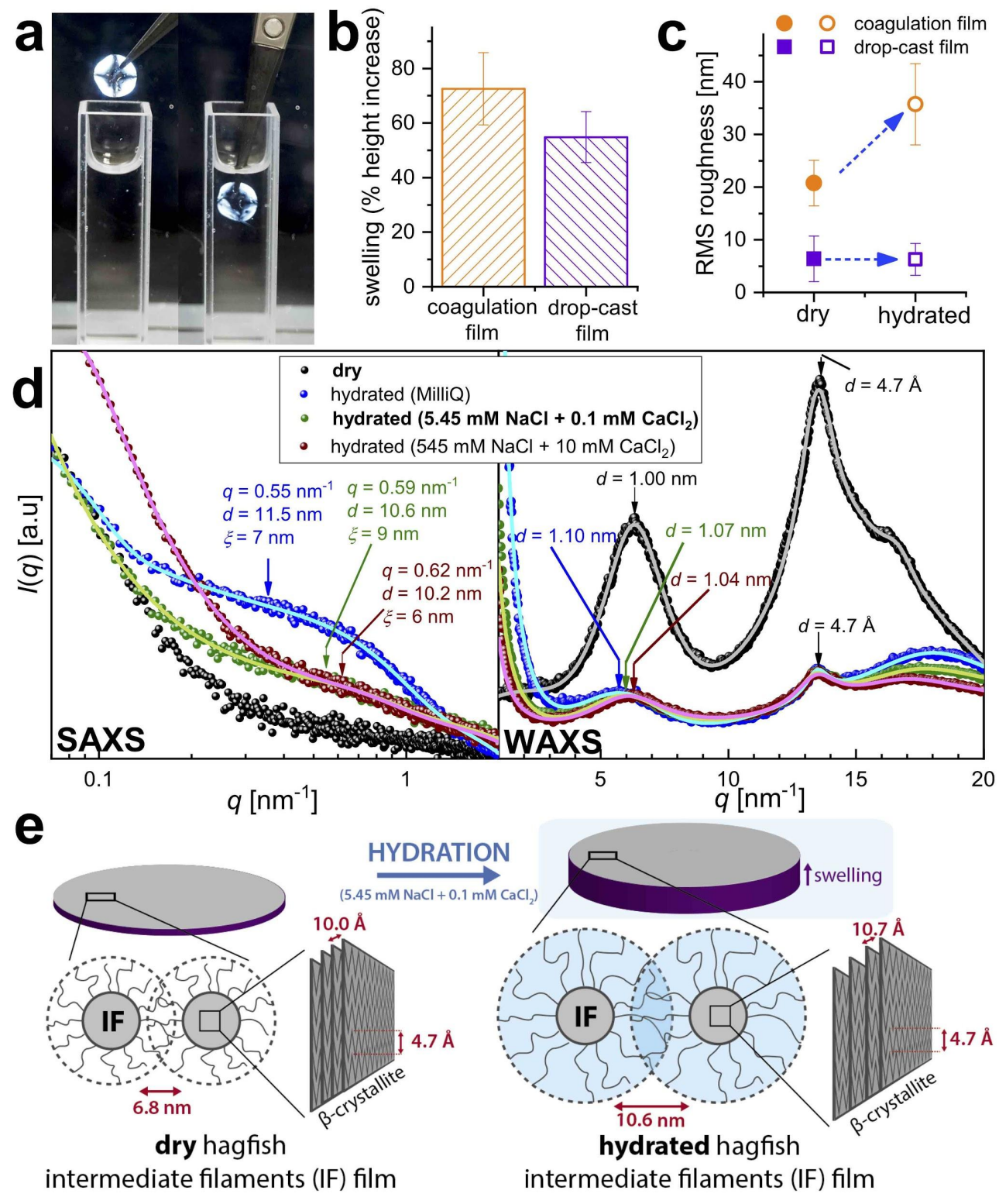
Figure 4: Hydration of intermediate filament protein films. (a) Hydration of a drop-cast film between cross-polarizers, showing birefringence for the dry and the hydrated state. (b) Swelling of the hydrated films, depicted as the height change percentage compared to the dry films. (c) Change in surface root mean square (RMS) roughness upon hydration (data based on images shown in Figure 3 and Figure S3). (d) Small angle (SAXS) and wide angle (WAXS) X-ray scattering profiles of dry and hydrated drop-cast films. For the hydrated films different salt concentrations were measured, whereby $5.45 \mathrm{mM} \mathrm{NaCl}+0.1 \mathrm{mM} \mathrm{CaCl} 2$ are the salt concentrations also used in AFM measurements in liquid. (e) Model depicting the hydration of hagfish IFs. The films are made of randomly oriented IFs. The amorphous C-terminal tail domains radiate perpendicular to the IF cylinder axis in a bottlebrush configuration. In the illustration the tail domains are end-grafted to a plane rather than a filament cylinder for simplicity. Upon hydration, the tail polymers stretch out and the brush height grows with polymer length.

X-ray measurements further suggest a polyelectrolyte behavior of hagfish IFs, which can be seen in the decrease of the average mesh-size $d$ and of the inter $\beta$-sheet distance upon salt addition (Figure 4d). The mesh-size decreased from $d=11.5 \mathrm{~nm}$ in Milli-Q to $d=10.6 \mathrm{~nm}$ in $1 \%$ SSW to $d=10.2 \mathrm{~nm}$ in $100 \%$ SSW, implying that charge screening effects at high salt levels reduce the expansion of the IF tail domains and thus probably cause the IF film to swell less. Polyelectrolyte properties of IFs are well known, especially for neurofilaments (NFs) as they are considered to regulate the steric and electrostatic repulsions between the amorphous sidearms and thus affect both single NFs as well as NF networks ${ }^{64-69}$ Also, hagfish thread skeins were found to display salt dependent properties ${ }^{28,70}$ Threads uncoiled substantially faster when subjected to Milli-Q compared to seawater, which was considered to result from an increased swelling of the hagfish fiber in Milli- $Q^{71}$

A mesh-size for the dry films can be estimated by relating the average mesh-size of a hydrated film $(d=10.6 \mathrm{~nm})$ to its macroscopic swelling ratio $V / V_{0}$. Using the swelling ratio of 1.55 for the drop cast film, an average mesh-size of $d=6.8 \mathrm{~nm}$ for the dry film is obtained. These results suggest that the entropic brush of tail domains, which maintain the inter-filament spacing expands from $6.8 \mathrm{~nm}$ to $10.6 \mathrm{~nm}$ upon hydration in $1 \%$ SSW, and thus causes swelling in hagfish IF films ${ }^{64,72}$. A schematic model depicting the structural changes occurring during hydration of hagfish IF films is shown in Figure 4e.

\section{Elastic modulus of dry and hydrated films}

Mechanical properties of the films were assessed using AFM nanoindentation. Representative force-distance (F-D) curves in air (dry films) and in water (hydrated films, in 1\% simplified seawater) with the respective Hertz fits to obtain the Young's modulus are shown in Figure 5 a. The reported elastic moduli in this study are referred to as 'apparent', as a result of the sudden load during indentation. Dry films had high apparent elastic moduli $\left(E \approx 10^{9} \mathrm{~Pa}\right)$, which

decreased about thousand fold upon hydration $\left(E \approx 10^{6} \mathrm{~Pa}\right.$ ) (Figure $5 \mathrm{~b}$ ). A thousand fold decrease in stiffness upon hydration is in line with observations for other matrix-free soft keratins such as stratum corneum and hagfish fibers ${ }^{35}$. The drop-cast films had a higher elastic 
modulus than the coagulation films, in both dry and hydrated state, which could be due to its increased $\beta$-sheet content and its higher density as the coagulation films appear porous i.e. are less dense. The elastic moduli of the dry films (5.1 GPa for coagulation films; 7.7 GPa for dropcast films) agree well with the value reported for dry hagfish fibers in extension $\left(E \approx 8 \mathrm{GPa}^{73}\right)$, and are in the range of elastic moduli determined by indentation for other dry keratins such as the cortical component of wool $\left(4 \mathrm{GPa}^{74}\right)$, hair $\left(7.4 \mathrm{GPa}^{75}\right)$, or fingernails $\left(4.6 \mathrm{GPa}^{76}\right.$. Furthermore, the elastic moduli of the hydrated films ( $E=6.1 \mathrm{MPa}$ drop-cast films; $E=3.9 \mathrm{MPa}$ coagulation films) agree well with elastic moduli reported for hydrated hagfish fibers $(6 \mathrm{MPa})^{24}{ }_{2}$ as well as for other IFs such as vimentin $\left(9 \mathrm{MPa}^{77}\right)$ but are a bit lower than values determined for single vimentin IFs in extension $\left(27 \mathrm{MPa}^{16}\right)$.

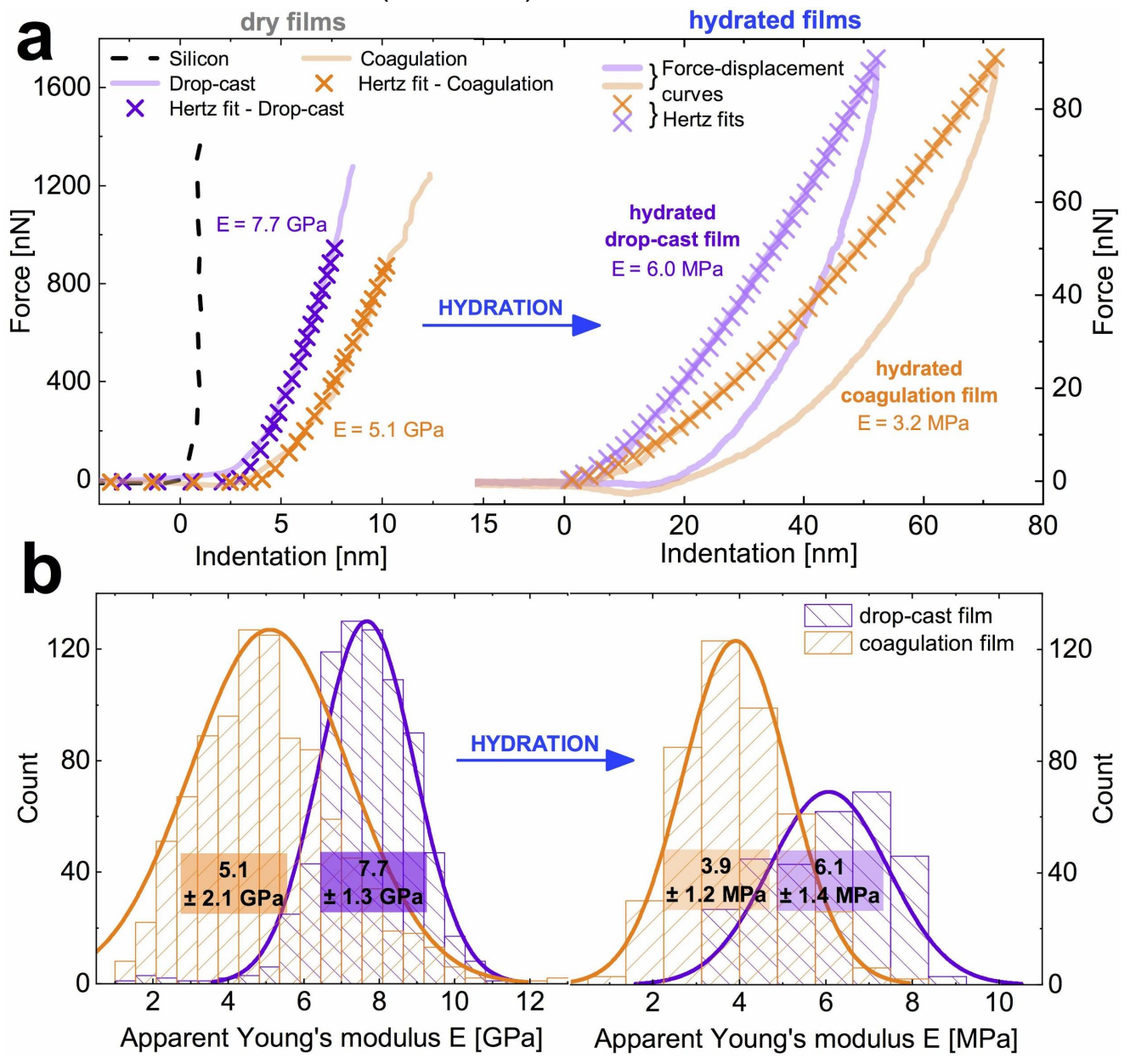

Figure 5: AFM force-distance (F-D) curves obtained from spherical indenters on dry and hydrated intermediate filament films. (a) Representative F-D curves obtained by indenting dry (left) and hydrated (right) hagfish protein films. The F-D were fitted with the Hertz model using the approach curve (hydrated films) and the retrace curve (dry films) to obtain an apparent Young's modulus. (b) Histograms depicting the elastic modulus $E$ from dry and hydrated films.

\section{Viscoelasticity of hydrated IF films}


Apparent elastic moduli were obtained using the Hertz fit, assuming a purely elastic response of the deformed material. This criterion was satisfied for the dry films, but the F-D curves on the hydrated films clearly showed energy dissipation, recognizable by a hysteresis loop between the approach and retraction curve (Figure 5a). Hysteresis was similarly observed in uniaxial extension experiments with hydrated hagfish fibers ${ }^{24}$. The hysteresis loops did not show fatigue after repeated indentation (Figure S4), implying that there is no permanent plastic deformation. Therefore, a behavior similar to tough hydrogels ${ }^{78}$ as well as viscoelasticity ${ }^{79,80}$ were observed. Viscoelasticity is well-known for IFs ${ }^{38,77,81}$ and indentation curves showing a similar recoverable hysteresis were observed for hydrated collagen fibrils ${ }^{82}$ and stratum corneum ${ }^{83}$, cells ${ }^{84}$, as well as polyacrylamide, PVA hydrogels ${ }^{84-86}$, and $\mathrm{PDMS}^{87,88}$. In order to assess the viscoelastic behavior of the hydrated IF films, two different approaches were used. On the one hand, constitutive viscoelastic parameters were extracted directly from AFM F-D curves using a method developed by Efremov et al. ${ }^{84}$, where the indentation speed was varied (Figure 6). On the other hand, viscoelastic properties were assessed from stress-relaxation measurements (Figure 7), where the effect of maximum load was investigated.
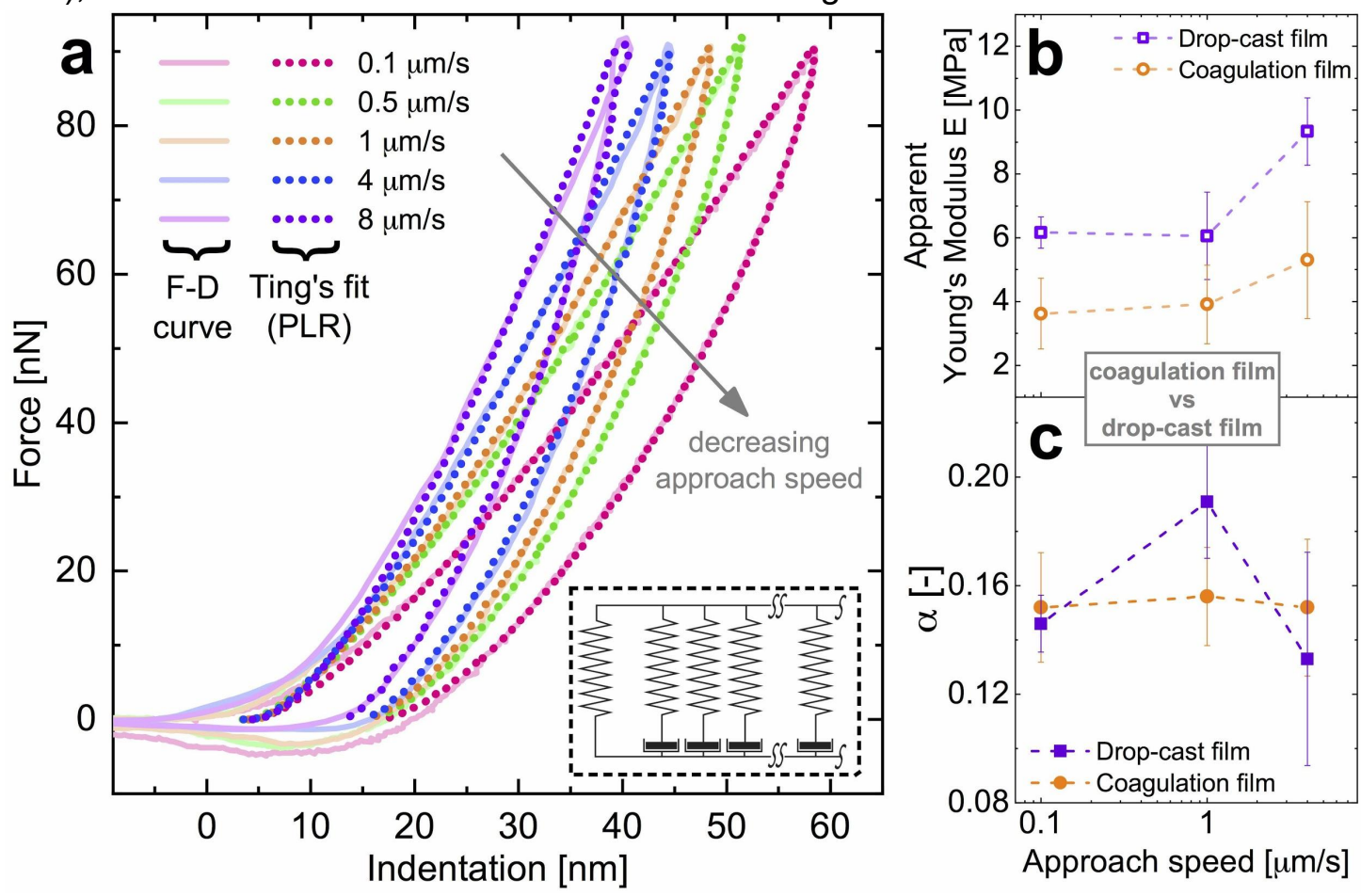

Figure 6: Effect of cantilever approach speed and cantilever size on viscoelastic properties of hydrated hagfish IF films. (a) Force-distance (F-D) curves on a hydrated coagulation film. The respective power-law rheology (PLR) fits using Ting's model ${ }^{89}$ are overlaid (dotted lines). The inlet shows a schematic drawing of the modified PLR model depicting the serial connection of spring-and-dashpot elements. (b) Effect of cantilever approach speed on the apparent elastic modulus. (c) Effect of cantilever approach speed on the power-law exponent $\alpha$ for a coagulation and drop-cast film. Every point in (b) and (c) represents an aggregation of a minimum of 120 force curves acquired by force-volume mapping. 
Increasing the cantilever approach speeds lead to a decrease in indentation depth (from approx. $60 \mathrm{~nm}$ at $0.1 \mu \mathrm{m} / \mathrm{s}$ to $40 \mathrm{~nm}$ at $8 \mu \mathrm{m} / \mathrm{s}$ ) in F-D measurements (Figure 6a) and caused an increase in apparent elastic moduli of roughly $50 \%$ (Figure $6 \mathrm{~b}$ ), both evidence for viscoelasticity ${ }^{88,90}$. The apparent elastic moduli at a cantilever approach speed of $0.1 \mu \mathrm{m} / \mathrm{s}$ (3.6 $\pm 1.1 \mathrm{MPa}$ for coagulation films; $6.1 \pm 0.5 \mathrm{MPa}$ for drop-cast films) increased about $50 \%$ when the speed was 40 fold increased to $4 \mu \mathrm{m} / \mathrm{s}(5.3 \pm 1.8 \mathrm{MPa}$ for coagulation films; $9.3 \pm 1.1 \mathrm{MPa}$ for drop-cast films). The power-law rheology (PLR) model (in depth explained in the methods section) fitted the F-D curves well over the range of investigated cantilever approach speeds and showed a predominantly soft-solid response, revealed by a power-law exponent $\alpha$ - where $\alpha=0$ means a solid-like and $\alpha=1$ a fluid-like behaviour. The power-law exponents of both films ( $\alpha=0.19 \pm 0.02$ for drop-cast films; $\alpha=0.16 \pm 0.02$ for coagulation films) were similar and did not vary substantially with speed. The derived instantaneous moduli $E_{0}(22 \pm 3 \mathrm{MPa}$ for drop-cast films; $12 \pm 3 \mathrm{MPa}$ for coagulation films) were higher than the apparent elastic moduli for both films, which could infer an underestimation of elasticity at very short experimental times. At a speed of $8 \mu \mathrm{m} / \mathrm{s}$ the F-D curves showed a slight 'twist' at peak indentation, suggesting artifacts caused by hydrodynamic effects or limitations in the AFM feedback loop due to the constant sampling rate of $2 \mathrm{kHz}$.
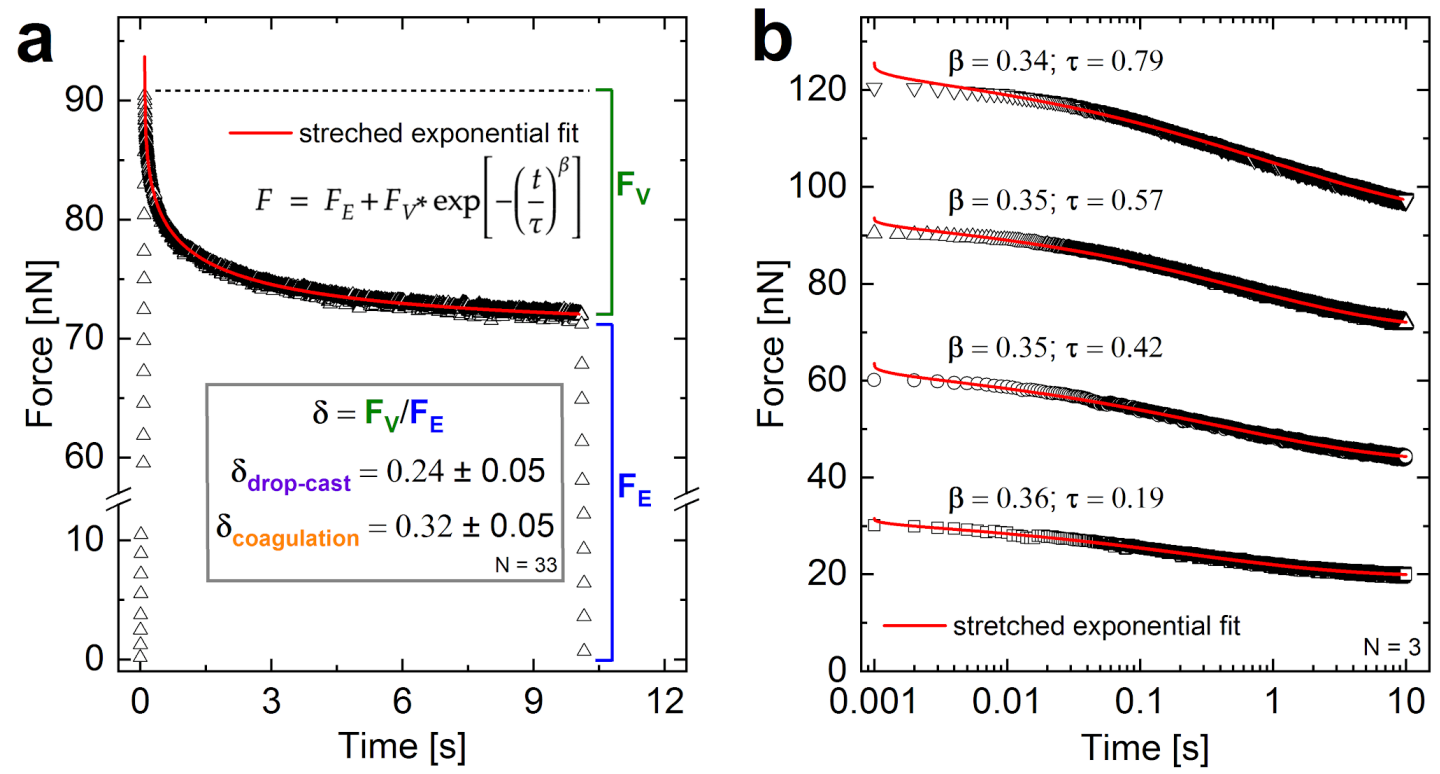

Figure 7: AFM stress-relaxation measurements on a hydrated hagfish IF film and the corresponding stretched exponential fits (red lines). (a) Stress relaxation of a coagulation film from an initial load of about $90 \mathrm{nN}$. The inlet depicts the calculation for $\delta-$ a parameter to relate elastic $F_{\mathrm{E}}$ and viscous $F_{\mathrm{V}}$ forces similar to the rheological loss factor tan $\delta$. (b) Stress relaxation measurements performed at increasing initial peak load (strain).

The stretched exponential model fitted the relaxation behavior of both hydrated IF films well (Figure 7a), yielding a fractional power-law exponent $\beta=0.45 \pm 0.07$ and a relaxation time $T=$ $0.40 \pm 0.07 \mathrm{~s}$ for the drop-cast film and $\beta=0.31 \pm 0.03$ and $\tau=0.75 \pm 0.22 \mathrm{~s}$ for the coagulation film. The lower $\beta$ for the coagulation film suggests a broader distribution of underlying relaxation 
times ${ }^{91}$, which could be due to its more heterogeneous and rough structure. Its heterogeneous and presumably less dense structure could also be a reason for the higher relaxation time $\tau$. Small discrepancies between the fit and the measurement data can be observed at very short times, which probably stem from experimental difficulties such as simultaneously detect short and long times and the challenge to impose an instantaneous step $\operatorname{strain}^{92}$. A parameter $\delta=$ $F_{\mathrm{V}} / F_{\mathrm{E}}$ was defined that relates the stored elastic $F_{E}$ to dissipated viscous stresses $F_{V}$ - similar to the loss factor tan $\delta=E^{\prime} / E$ in rheology - was calculated from the relaxation measurements (Figure 7a, inlet). Drop-cast films showed a $\delta=0.24 \pm 0.05$, suggesting that they retain more stored elastic stresses than the coagulation films $(\delta=0.32 \pm 0.05)$ after $10 \mathrm{~s}$ relaxation corresponding to a theoretical oscillatory frequency of $0.1 \mathrm{~Hz}$. These values supports the finding that hydrated hagfish IF films show viscoelastic solid-like behavior and dissipate viscous stresses under load. Increasing loads in stress relaxation measurements did not affect the stretch exponent $\beta$ but resulted in increased relaxation times $T$ (Figure $7 \mathrm{~b}$ ). This implies that strain (in the tested range) does not affect the overall relaxation mechanism of the many independently relaxing structures. However, higher strains increase the time these structures require to relax, implying the relaxation of physical bonds rather than chemical cross-links. The more physical entanglements are stressed the more time they require to dissipate this stress. The PLR model also fitted the stress relaxation measurements well and yielded similar powerlaw exponents $(0.05<\alpha<0.2)$ for increasing step loads (Figure S5) as obtained from the F-D curves using Ting's model. However, the less parametrized stretched-exponential model was found more resilient to describe the stress-relaxation behavior. The challenge to impose an instantaneous step load especially for AFM in liquid environments as well as the possibility that residual stresses in the bent cantilever co-relax with the hydrated film during the long dwells (10 s) and thus cause a superimposed relaxation - which is less the case in the faster F-D measurements - seem to affect the PLR model more and therefore the stretched-exponential model was chosen to describe the stress-relaxation in the dwell measurements.

Based on the mechanical and structural findings, we propose that hagfish IF films possess an amorphous matrix constituted by the IF tail domains with embedded $\beta$-crystallites, originating from the central part of the IFs. It is challenging to distinguish whether the structure is a connective $\beta$-crystallite matrix with amorphous domains or a connective amorphous matrix with embedded $\beta$-crystallites only from FTIR and X-ray measurements. However, the thousand-fold reduction in elasticity upon hydration strongly favours latter model, as a $\beta$-crystallite matrix is unlikely to soften this substantially. Furthermore, the finding that larger strains require more time for the system to relax suggests that the contact points in hagfish IF films are the physical, which is likely due to the entangled and overlapping IF tail domains.

\section{Hydrated IF films do not exhibit strain stiffening}

It was further tested if increasing strain provokes strain stiffening in hydrated IF films in indentation measurements (Figure S6). Spanning a range between 20 - $250 \mathrm{~nm}$ indentation depth no strain stiffening was observed. The apparent elastic modulus, as well as the power-law exponent $\alpha$ from the PLR fit remained constant except for the smallest indentation, and when the indenter was changed from a spring constant of $1.75 \mathrm{nN} / \mathrm{nm}$ to $38.5 \mathrm{nN} / \mathrm{nm}$ (Figure S6) in order to achieve larger indentations and still operate in the linear range of cantilever deflection. It is likely that surface roughness of the coagulation film as well as the small cantilever 
deflection caused inaccuracies at the smallest indentation depth. The small change of moduli upon cantilever change could be due to minor deviations in the deflection calibration of the cantilever. Strain stiffening was described for IF hydrogel networks ${ }^{63,77,81,93}$ and is caused by an entropy loss associated with the stretching of filaments in between cross-links of the (hydrogel) network $^{63}$. For strain hardening to occur the contact points must be sufficiently strong in order to stretch the filament strands as otherwise the links break and the strands relax ${ }^{93,94}$. The contact points (i.e., overlapping and entangling IF tail domains) in hagfish IF films likely keep the films together, prevent dissolution, and are mainly responsible for the viscoelastic response. However, as only $\beta$-sheets are observed, it is possible that the increased rigidity of $\beta$-sheets compared to the more flexible $\alpha$-helical coiled-coils ${ }^{95,96}$ prevents stretching of the IF. The physical contact points of the 'tail-domain matrix' might be too weak to stretch the $\beta$-sheets. Furthermore, it is possible that indentation is less prone to induce strain stiffening as it exerts compression rather than stretching. Compression might require higher strains, i.e., deeper indentations in order to achieve the same amount of stretching than shear or extension.

\section{Implications of viscoelasticity for biomimetic fiber production}

Coagulation films can be lifted up from the water interface of the $\mathrm{MgCl}_{2}$ buffer and pulled into macroscopic fibers ${ }^{36}$ (Figure 8a). The fibers reached lengths up to $12 \mathrm{~cm}$, which is about five times longer than reported previously ${ }^{32}$ and in the range of natural hagfish fibers $(10-20$ $\mathrm{cm}^{28,29}$ ). The hydrated film formed a 'curtain' that is stretched at the air-water interface upon pulling (Figure $8 \mathrm{~b}$ ). The stretching caused an alignment of the otherwise randomly distributed IFs along the fiber axis (Figure 8c) similar to natural hagfish fibers (Figure 8d) as shown by WAXS measurements. The WAXS pattern of the reconstituted fibers (inter $\beta$-strand spacing of $0.46 \mathrm{~nm}$; inter $\beta$-sheet spacing of $0.94 \mathrm{~nm}$ ) strongly resembled the patterns of natural stretched hagfish fibers (inter $\beta$-strand spacing of $0.46 \mathrm{~nm}$; inter $\beta$-sheet spacing of $0.94 \mathrm{~nm}$ ) and also showed a silk-like alignment of the $\beta$-crystallites along the fiber axis as reported for IFs in natural hagfish fibers ${ }^{17,24}$. The alignment of the IF $\beta$-crystallites was assessed by the order parameter $S$ (Figure 8e,f), which provides a measure of the long-range crystalline order of a material and is defined as the second Legendre polynomial $\left.S=\frac{1}{2}<3 \cos ^{2} \phi-1\right\rangle$, where $\phi$ is the angle between the axis of an individual molecule and the director of the crystal averaged over the complete ensemble ${ }^{97}$. The order parameter ranges from a perfect parallel orientation $(S$ $=1$ ) to a perfect perpendicular orientation $(S=-0.5)$ and is $S=0$ for unoriented material ${ }^{98,99}$. For both the reconstituted and the natural fibers order parameters of $S \approx 0.3$ in the low q-range (15 $\left.\mathrm{nm}^{-1}<\mathrm{q}<17 \mathrm{~nm}^{-1}\right)$ and $S \approx 0.5$ in the high q-range $\left(5.6 \mathrm{~nm}^{-1}<\mathrm{q}<7.6 \mathrm{~nm}^{-1}\right)$ were found, implying a nematic-like alignment of the $\beta$-strands (high q; from 15 to $17 \mathrm{~nm}^{-1}$ ) and $\beta$-sheets (low q, from 5.6 to $7.6 \mathrm{~nm}^{-1}$ ).

We suggest that a combination of viscoelasticity and the interaction of the film with the water interface are crucial for the production of such long biomimetic fibers with aligned $\beta$-sheets. The viscoelastic properties allow stretching and relaxation during stretching, which seems important to manufacture long fibers without removing the film from the surface at once. Furthermore, strong interactions probably due to hydrogen bonding between the film and the water interface are crucial to achieve a substantial stretching of the 'curtain' and thus create long fibers with 
aligned IFs. We found that tiny residues of surfactants in the buffer prevented the pulling of long fibers, underlining the importance of the interaction of the film with the water interface. Also, we found that in contrast to a previous work which found that a secondary drawing of the reconstituted fibers increased IF alignment ${ }^{32}$, IF alignment was only minimally improved by 'double drawing' in this work. This shows that if the film strongly interacts with the buffer surface, it is sufficiently stretched and thus most IFs are aligned already during pulling from the interface.

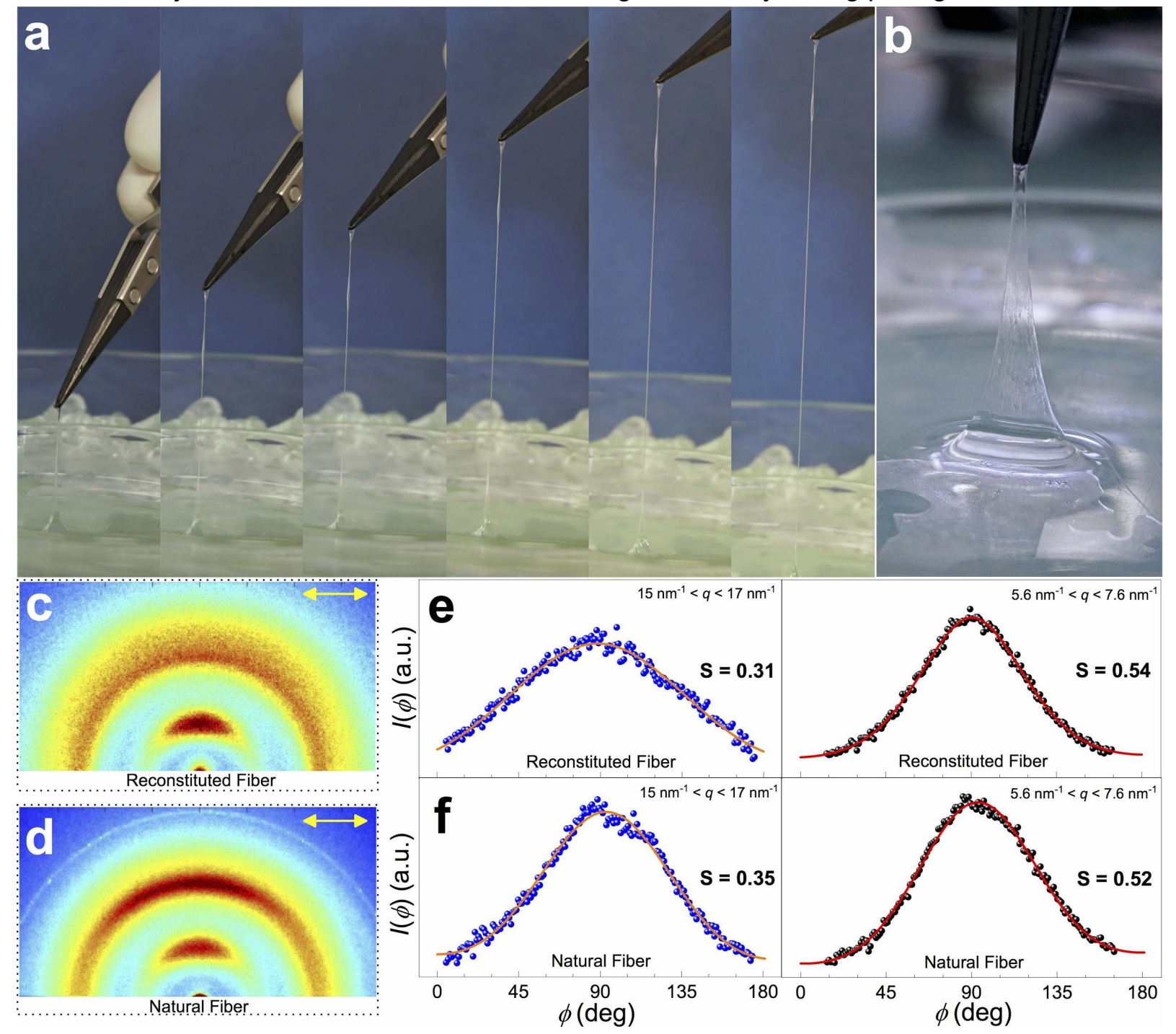

Figure 8: Implications of viscoelasticity on the production of biomimetic fibers. (a) Drawing a coagulation film from the $\mathrm{MgCl}_{2}$ buffer surface into a long fiber. (b) Close-up of the coagulation film 'curtain' during fiber pulling. The curtain is stretched at the water interface, causing the randomly distributed IFs to align with the fiber axis. (c,d) 2D WAXS patterns of reconstituted and natural hagfish fibers showing the axial alignment of $\beta$-sheets. The yellow arrow denotes the fiber axis direction. (e,f) Azimuthal intensity distribution of the WAXS profiles and the derived order parameter $S$, showing showing the silk-like alignment of the IF $\beta$-sheets in both the reconstituted and the natural hagfish fibers. 


\section{Conclusion}

Intermediate filament (IF) films from hagfish fiber protein were produced by drop-casting and coagulation on a $\mathrm{MgCl}_{2}$ buffer. Drop-casting yielded self-supporting, smooth, and dense films rich in $\beta$-sheets $(61 \%)$ whereas coagulation formed thin, porous films with a nano-rough surface and a lower $\beta$-sheet content $(51 \%)$. Both films were birefringent and can be considered semicrystalline nanocomposites, consisting of $\beta$-crystallites (IF central part) and random coils (IF tails). When immersed in water the films immediately swelled, resulting in a height increase of 55 and $73 \%$ in for the drop-cast and the coagulation film, respectively. Small and wide angle Xray scattering revealed that the $\beta$-crystallites remained stable upon hydration and that swelling presumably happens in the C-terminal tail-domains of the IFs. These amorphous tails are considered to be radially grafted to the IFs in a bottle-brush configuration, which hydrates and increases in diameter and thus causes swelling. X-ray measurements further revealed a polyelectrolyte behavior of hagfish IFs as the average mesh-size of the IF network as well as the inter $\beta$-sheet distance decreased upon increase of salt concentration due to charge screening, with Milli-Q showing a larger average mesh-size of $11.5 \mathrm{~nm}$ compared to simplified seawater (SSW) with an average mesh-size of $10.2 \mathrm{~nm}$.

Using AFM nanoindentation it was observed that hydration in $1 \%$ SSW caused a roughly thousand-fold decrease in apparent elastic modulus from $10^{9}$ to $10^{6} \mathrm{~Pa}$. The apparent elastic moduli for the drop-cast films $\left(E_{\text {dry }}=7.7 \mathrm{GPa}, E_{\text {hydrated }}=6.1 \mathrm{MPa}\right)$ were higher than those of the coagulation films $\left(E_{\text {dry }}=5.1 \mathrm{GPa}, E_{\text {hydrated }}=3.9 \mathrm{MPa}\right.$ ), which could be due to their higher $\beta$-sheet content and the lower density of the porous coagulation films. The hydrated films displayed distinct viscoelastic behavior characteristic for soft-solid and tough hydrogels identified by repeatable and fully recoverable hysteresis loops in force-distance (F-D) curves, velocity dependence of the elastic modulus and indentation depth, as well as partly viscous stress relaxation. Fitting a power-law rheology (PLR) model directly to the (F-D) curves yielded a power-law relaxation exponent $\alpha$ of roughly $<0.2$ for both films, suggesting $80 \%$ of elastic storage and $20 \%$ of viscous loss in force measurements, which was supported by stress relaxation measurements. Fitting a stretched exponential force decay model to stress relaxation measurements of hydrated IF films further revealed a lower stretched exponential factor $\beta$ for coagulation films $(\beta=0.31)$ than for drop-cast films $(\beta=0.45)$, implying a broader distribution of relaxation times for the coagulation films. Also, coagulation films exhibited a higher relaxation time $(\tau=0.75 \mathrm{~s})$ than drop-cast films $(\tau=0.4 \mathrm{~s})$ at a given peak load of $90 \mathrm{nN}$, implying that the more heterogeneous and porous structure takes longer to relax. Increasing loads (strain) in stress relaxation resulted in higher relaxation times, suggesting that physical entanglements are more disrupted at larger strains and thus take longer to dissipate the stress. We propose that hagfish IF films are semi-crystalline nanocomposites made from $\beta$-crystallites from an $\alpha \rightarrow \beta$

transformed central part of the IFs embedded in an amorphous matrix constituted by the physically entangled C-terminal tail-domains, which determines cohesion and viscoelasticity in hydrated films. Upon stress the matrix entanglements are disrupted whereby a larger stress causes more disruption and thus requires a longer time for the physical contact points to dissipate this stress. These physical contact points of the tail-domain matrix do not seem strong enough to stretch the IF $\beta$-sheets and thus induce strain hardening. 
We further suggest that viscoelasticity and strong hydrogen bonding interactions of the coagulation film with the buffer surface are crucial for a successful fiber making process, in which a coagulation film is pulled from the buffer interface into a fiber. The combination of relaxing stresses within the film and strong hydrogen bonding of the film with the water interface allow for a continuous stretching yet prevent early removal of the film from the interface, thus creating long biomimetic fibers with high IF alignment similar to natural hagfish fibers. X-ray measurements revealed that this method yields reconstituted fibers that possess order parameters of $S \approx 0.3$ for the $\beta$-strands and $S \approx 0.5$ for the $\beta$-sheets, implying a nematic-like alignment as similarly observed in natural fibers. This study shows that functional IF materials that immediately swell and soften in water without dissolving can be produced from hagfish slime fibers, which could potentially be used in applications such as contact lenses, scaffolds for cell cultures, smart textiles, food preservation, and non-lethal naval defense.

\section{Materials and Methods}

Exudate sampling and protein purification: Exudate sampling and storage in $1 \mathrm{M}$ citrate/PIPES (CP) buffer is in depth described by Böni et al. ${ }^{100}$. Exudate in CP buffer was concentrated by mild centrifugation to avoid compaction (1000 g, $5 \mathrm{~min}$ ). The supernatant containing large amounts of mucin vesicles was filtered through a series (60 and $20 \mu \mathrm{m}$ ) of nylon mesh filters (Merck) to separate remaining skeins from the vesicle suspension. The pelleted skeins were then washed in cycles comprising resuspension with CP buffer, mild centrifugation, and decanting. In the first three cycles the washing was done with CP buffer, then with CP buffer containing $10 \mathrm{mM}$ DTT, then with $0.5 \mathrm{M} \mathrm{CP}$ buffer, with $50 \mathrm{mM}$ DTT in Milli$Q$, and finally three times with Milli-Q only. The skeins and the mucin vesicle suspension were dialyzed against 3 I Milli-Q over $24 \mathrm{~h}$ at $4^{\circ} \mathrm{C}$ using a pre-wetted RC dialysis tube (MWCO 25 $\mathrm{kDa}$; Spectrum Laboratories, USA), comprising three water changes. The dialyzed samples were snap frozen in liquid $\mathrm{N}_{2}$ and freeze-dried (VirTis BenchTop Pro, SP Scientific, USA) for 24 h. The dry samples were stored airtight at room temperature until further use. Import of the samples was approved by the Swiss Federal Food Safety and Veterinary Office (FSVO) and export was approved by Norwegian Seafood Council.

Production of films and fibers: Two kinds of films were produced, 'drop-cast' and 'coagulation' films. A protein dope for film production was prepared by solubilizing lyophilized skeins in $\geq 96 \%$ formic acid for $1 \mathrm{~h}$ to obtain a $10 \% \mathrm{w} / \mathrm{v}$ stock solution ${ }^{36}$. Proper dissolution of the protein required intense vortexing during the first $10 \mathrm{~min}$. For drop-casting $50 \mu \mathrm{l} \mathrm{of} 5 \% \mathrm{w} / \mathrm{v}$ dope were pipetted on a flat teflon surface, forming a film with a diameter of about $8 \mathrm{~mm}$. The film was removed after drying $24 \mathrm{~h}$ at ambient air. Coagulation films were produced following a slightly modified protocol of Negishi et al. ${ }^{36}$. In brief, about $10 \mu \mathrm{l}$ of the $5 \% \mathrm{w} / \mathrm{v}$ protein dope were dropped on the surface of an ice cooled coagulation bath containing $100 \mathrm{mM}$ magnesium chloride $\left(\mathrm{MgCl}_{2}\right)$ and $20 \mathrm{mM}$ 4-(2-hydroxyethyl)-1-piperazineethanesulfonic acid (HEPES) at pH 7.5. Before film formation, a fine woven wire cloth with $1 \mathrm{~mm}$ mesh-size (Haver\&Boecker, Germany) and above it a thin teflon plate with holes (8 $\mathrm{mm}$ diameter) were immersed in the coagulation bath. The film formed in the hole of the teflon slide and was lifted out of the bath after $15 \mathrm{~s}$. The underlying metal mesh ensured that the film was not stretched by surface 
tension during removal. For fiber production the coagulation films were picked with tweezers in the center and lifted with a speed of approx. $2 \mathrm{~cm} / \mathrm{s} 15 \mathrm{~s}$ after formation. The fibers were placed on a flat teflon slide and dried at room temperature for $24 \mathrm{~h}$. Natural hagfish fibers for X-ray measurement were obtained by placing hagfish slime on a teflon plate and gently stretching the slime uniaxially. The aligned slime fibers were air dried for $24 \mathrm{~h}$, washed with $50 \mathrm{mM}$ DTT and Milli-Q and subsequently dried again for $24 \mathrm{~h}$.

Microscopy: Transmission light microscopy was performed on a Nikon Diaphot (Nikon, Japan), and cross polarizers were used for birefringence assessment. Images were analyzed with the NIS elements D3.0 software. Confocal laser scanning microscopy (CLSM) was performed on a Zeiss LSM 780 (Carl Zeiss AG, Germany). Slime fibers were stained with Nile Blue (Sigma Aldrich, USA) and imaged in reflection made. Freeze dried mucin was not stained and imaged in transmission made. Image stacks were acquired and collapsed to a yield 2D image.

Gel electrophoresis: $200 \mu \mathrm{g}$ freeze dried skeins were mixed with $100 \mu \mathrm{l} 1 \mathrm{x}$ Laemmli loading buffer. The samples were ultrasounded for $10 \mathrm{~min}$ at frequency of $80 \mathrm{kHz}$ (Emasonic P, Elma, Germany) and subsequently heated for $15 \mathrm{~min}$ at $90^{\circ} \mathrm{C}$ with a mixing rate of $500 \mathrm{rpm}$ (Mixing Block MB102, ACTGene, USA). Before loading, all samples were centrifuged at $10^{\prime} 000 \mathrm{~g}$ (Biofuge Pico, Heraeus, Germany) to remove non-solubilized cellular debris. The gels were run on 10-20 \% Criterion TGX Stain-Free Precast Gels (Bio-Rad Laboratories, USA). The reactor was filled with electrophoresis buffer, which was diluted from a $5 \times$ stock solution $(0.25 \mathrm{M}$ TRIS, $1.92 \mathrm{M}$ glycine and $0.5 \%$ SDS in Milli-Q). $10 \mu \mathrm{l}$ protein ladder (Precision Plus Protein Standards Dual Color, Bio-Rad Laboratories, USA) were loaded along with $20 \mu \mathrm{l}$ of sample. The sample was run for $20 \mathrm{~min}$ at $100 \mathrm{~V}$ and subsequently for $12 \mathrm{~min}$ at $300 \mathrm{~V}$. The gel was stained for $1 \mathrm{~h}$ (0.05\% w/v Coomassie blue R250, $50 \%$ v/v methanol, $10 \%$ v/v acetic acid glacial and $40 \%$ $\mathrm{v} / \mathrm{v}$ Milli-Q) and subsequently destained (10\% v/v methanol, $7.5 \% \mathrm{v} / \mathrm{v}$ acetic acid glacial and $82.5 \% \mathrm{v} / \mathrm{v}$ Milli-Q) on an agitation unit (Automated Gel Stainer, Hoefer, USA) for $24 \mathrm{~h}$. The destaining solution was replaced several times. An image of the gel was acquired using the Molecular Imager $($ Gel DocTM XR+ System with Image LabTM Software 5.2.1 (Bio-Rad Laboratories, USA). The molecular weight of the bands was calculated according to the linear equation of the logarithmic weight of the protein marker versus the running distance relative to the dye front.

ATR-FTIR: Attenuated total reflectance - Fourier transform infrared spectroscopy (ATR-FTIR) was performed on an FTIR device (Varian 800 FTIR, Varian, USA) equipped with an ATR accessory (Golden Gate Standard, Specac, USA). The software Resolutions (Resolutions 4.0, Varian, USA) was used for acquisition of the ATR-FTIR spectra. The spectra were acquired in the wavenumber range of $600-4000 \mathrm{~cm}^{-1}$ with a resolution of $4 \mathrm{~cm}^{-1}$. The number of scans was set to 64 and automatic atmospheric compensation was performed. The background was subtracted from all spectra. For qualitative comparison, the spectra were normalized between 0 and 1 by the maximum of the amide I peak. Peak deconvolution was performed with the software OriginPro 9.1 .0 (Electronic Arts, USA). The amide I peak $\left(\sim 1650 \mathrm{~cm}^{-1}\right)$ was selected visually and the edges were defined as local minima on each side of the peak. A straight line was subtracted to correct the tilt of the amide I peak. The second derivative with a SavitzkyGolay smoothing was calculated with a polynomial order of 2 and 7 points of window. The peak 
deconvolution was performed using a multiple Pseudo-Voigt I peak fit and by selecting the minima of the second derivative, indicating the location of the deconvolved peaks. All fits had an $R^{2}$ of $>0.99$. Choice of peak assignments to secondary structure motifs is based on the work of Hu et al. ${ }^{58}$ and Zou et al. ${ }^{101}$.

X-ray diffraction (XRD): Small angle X-ray scattering (SAXS) and wide angle X-ray scattering (WAXS) experiments were performed at ambient temperature. Fibers: WAXS experiments on fibers were performed using a Bruker AXS Micro (Bruker, USA) equipped with a microfocused beam $(50 \mathrm{~W}, 50 \mathrm{kV}, 1 \mathrm{~mA})$ with the $\mathrm{I}_{\text {CuKa }}=0.15418 \mathrm{~nm}$ radiation in order to obtain direct information on the scattering patterns. The scattering intensities were collected by a twodimensional Dectris 2D Pilatus $100 \mathrm{~K}$ X-ray detector $(83.8 \mathrm{~cm} \times 33.5 \mathrm{~cm}, 172 \mu \mathrm{m}$ resolution) (Dectris, Switzerland). An effective scattering vector range of $1 \mathrm{~nm}^{-1}<\mathrm{q}<25 \mathrm{~nm}^{-1}$ was obtained, where $q$ is the scattering wave vector defined as $q=4 \pi \sin \theta / I_{\text {cuka }}$ with a scattering angle of $2 \theta$. Swollen and dry films: SAXS and WAXS experiments on dry and swollen films were performed using a Rigaku MicroMax- $002^{+}$(Rigaku, Japan) equipped with a microfocused beam $(40 \mathrm{~W}, 45 \mathrm{kV}, 0.88 \mathrm{~mA})$ with the $\mathrm{I}_{\text {CuKa }}=0.15418 \mathrm{~nm}$ radiation collimated by three pinhole collimators $(0.4,0.3$, and $0.8 \mathrm{~mm})$ in order to obtain direct information on the scattering patterns. The SAXS and WAXS intensities were collected by a two-dimensional Triton-200 gas-filled Xray detector (20 cm diameter, $200 \mu \mathrm{m}$ resolution) and a two-dimensional Fujifilm BAS-MS 2025 imaging plate system (15.2 × $15.2 \mathrm{~cm}^{2}, 50 \mu \mathrm{m}$ resolution) (Fujifilm, Japan), respectively. An effective scattering vector range of $0.05 \mathrm{~nm}^{-1}<\mathrm{q}<25 \mathrm{~nm}^{-1}$ was obtained. WAXS intensity profiles were deconvoluted by using pseudo-Voigt peak functions. Hydrated films were evaluated by using a gel-like model including a pseudo-Voigt peak function, where the resulting peak position is related to the average distance between solvent pockets (mesh-size).

AFM imaging and nanoindentation: AFM (atomic force microscopy) was performed on a Cypher (Asylum Research, USA). Images were acquired in tapping mode in air and water using OMCL-AC240TS cantilevers (Olympus, Japan). In water the cantilevers were driven with the Blue Drive ${ }^{\circledR}$ technology. Nanoindentation was performed using gold-coated high-precision spherical tip cantilevers (Nanotools, Germany) made of diamond-like carbon. The tip radius of each cantilever is measured in high resolution SEM and is precise down to the nanometer. Dry films were indented in air using a $50 \mathrm{~nm}$ radius indenter (B50-NCAu) with a spring constant of $32 \mathrm{nN} / \mathrm{nm}$ at $2 \mu \mathrm{m} / \mathrm{s}$. Indentation in liquid was performed using indenters with $500 \mathrm{~nm}$ radius (B500-NCAu and B500-FMau) with spring constants of $38.5 \mathrm{nN} / \mathrm{nm}$ and of $1.75 \mathrm{nN} / \mathrm{nm}$, respectively. Unless stated differently indentation in liquid was performed at $1 \mu \mathrm{m} / \mathrm{s}$ approach speed. The thermal method was used to determine the spring constant of the cantilevers. Before every indentation measurement, the InvOLS were determined by indenting on silica substrate. Force-volume maps were acquired containing between 120 - 1200 force curves. Drop-cast films were directly cast on silicon substrate and dried for $24 \mathrm{~h}$ at room temperature. As discussed in the results part, casting on glass - as well as on silicon - leads to a $\beta$-sheet orientation parallel to the film surface, resulting in homogeneous mechanical properties throughout the film. Washed and dry coagulation films were placed on silicon substrates and gently hydrated with Milli-Q, which caused the films to stick and allowed subsequent repeated hydration without removal. All measurements were performed at room temperature.

Film hydration: The films were hydrated in deionized water (Milli-Q), in simplified seawater (SSW) $\left(545 \mathrm{mM} \mathrm{NaCl}+10 \mathrm{mM} \mathrm{CaCl}_{2}\right)$ to mimic the high salt conditions faced by natural hagfish 
fibers as well as in $1 \% \mathrm{SSW}\left(5.45 \mathrm{mM} \mathrm{NaCl}+0.1 \mathrm{mM} \mathrm{CaCl}_{2}\right)$. For AFM nanoindentation and swelling height measurements the films were hydrated in 1\% SSW in order to screen surface charges. Swelling of films was assessed with AFM using force-volume mapping (FVM). Similar to imaging in tapping mode, FVM provides a height profile by determining the contact point of cantilever and sample. The combination of this 'low speed tapping' with the long approach/retrace distances avoids dragging of the sample and allows to image the same sample in air and subsequently in liquid without changing sample, or cantilever. Thus, the exact same position can be monitored before and after hydration. All FVM images were acquired on a sample edge (scratched with a scalpel) in order to always have both, sample and substrate on the same image to more accurately monitor swelling. The height images were flattened (1st order) and the difference between the medium value of the height histogram of the film to the histogram of the substrate was recorded as height. All measurements were performed on three different films at room temperature.

Roughness calculation: The root mean square (RMS) roughness of the films was calculated from $5 \times 5 \mu \mathrm{m}$ AFM images, which were segmented into $251 \times 1 \mu \mathrm{m}$ squares (Table S1) in order to obtain RMS roughness values within the relevant range for indentation (indenter size = $1 \mu \mathrm{m}$ diameter in water). The images were flattened (0 order) and the RMS roughness was calculated using Igor Pro (WaveMetrics, USA).

Calculation of apparent elasticity: The apparent Young's modulus was extracted by fitting Hertz contact model (eq. 1) to the loading part of the hydrated films and to the unloading part of the dry films of the AFM force-distance (F-D) curves using Igor Pro (WaveMetrics, USA) and Matlab (Mathworks, USA). The Hertz model for a spherical indenter has the following form:

$$
F(\delta)=\frac{4 \sqrt{R}}{3\left(1-\nu^{2}\right)} E_{H e r t z} \delta^{3 / 2}
$$

Equation 1

,where $F$ is the force acting on the cantilever, $\delta$ is the indentation depth, $R$ is the radius of the spherical indenter, $E_{\text {Hertz }}$ is the Young's modulus, and $v$ is the sample Poisson's ratio (assumed to be 0.5 for full volume conservation).

Calculation of viscoelasticity: Viscoelastic parameters of the hydrated films were obtained on the one hand i) directly from F-D curves using an approach by Efremov et al. ${ }^{84}$ and on the other hand by ii) fitting a stretched exponential model to stress-relaxation measurements. All (visco)elasticity values were corrected for bottom effects (finite sample thickness) where required ${ }^{102,103}$.

i) Viscoelasticity directly from F-D curves: The major challenge in viscoelastic analysis of F$D$ curves in AFM nanoindentation testing is a moving boundary condition. Pushing a hard indenter into a soft viscoelastic material results in a changing contact area with time between the indenter and the sample half-space, making the elastic-viscoelastic correspondence principle not applicable ${ }^{104}$. Lee and Radok $^{105}$ showed that if the solution of the elastic problem is known, then the time-dependent stresses and deformations for an axisymmetric indenter (i.e. the solution to the corresponding viscoelastic problem) may be found by replacing the elastic modulus after introduction of an appropriate hereditary integral operator ${ }^{84,104}$. Their method provides a time-dependent indentation depth for any load history that does not produce a decrease in contact area, i.e. the function is only valid for the approach curve. ${ }^{84}$. A more general approach to solving the linear viscoelastic contact problem for an approach-retraction 
indentation cycle with a spherical rigid indenter was later presented by Ting ${ }^{89}$. Ting's solution can be applied to any arbitrary history of contact area and is described by following equations:

$$
\begin{aligned}
F(t, \delta(t))=\left\{\begin{array}{cc}
\frac{4 \sqrt{R}}{3\left(1-\nu^{2}\right)} \int_{0}^{t} E(t-\xi) \frac{\partial \delta^{3 / 2}}{\partial \xi} \partial \xi, & 0 \leqslant t \leqslant t_{m} \\
\frac{4 \sqrt{R}}{3\left(1-\nu^{2}\right)} \int_{0}^{t_{1}(t)} E(t-\xi) \frac{\partial \delta^{3 / 2}}{\partial \xi} \partial \xi, \quad t_{m} \leqslant t \leqslant t_{\text {ind }} & \text { Equation 2 } \\
\int_{t_{1}(t)}^{t} E(t-\xi) \frac{\partial \delta}{\partial \xi} \partial \xi=0 & \text { Equation 3 }
\end{array}\right.
\end{aligned}
$$

Where $E(t)$ is the Young's relaxation modulus (analogous to the sample relaxation function of Lee and Radok), $t$ is the time of initial contact between sample and indeter, $t_{m}$ is the duration of the approach phase, $t_{\text {ind }}$ is the duration of the complete indentation cycle) and $t_{1}$ is the auxiliary function (Eq. 3). The first part of Equation 2 is valid for the indentation approach curve, during which the contact area increases ( $0 \leq t \leq t_{m}, t_{m}$ is the end of the approach phase). This solution is equal to the solution presented by Lee and Radok. The second part of Equation 2 is valid for the retraction curve $\left(t>t_{m}\right)$. Here, an auxiliary function $t_{1}$ is introduced and defined by $a\left(t_{1}\right)=a(t)$, $t_{1}(t)<t_{m}$, which means that the contact area $a$ at time $t$ during the retraction phase is equal to the contact area at time $t_{1}$ during the approach phase. The $t_{1}$ values found must satisfy the formula in the Equation 2. Using the auxiliary $t_{1}(t)$ function, the contact area $a\left(t_{1}(t)\right)$ and the effective indentation $\delta_{1}\left(t_{1}(t)\right)$ during the retraction phase - best imagined as the indentation relative to the relaxing sample surface - can then be calculated. A time-dependent relaxation modulus $E(t)$ then allows for the use of linear viscoelastic constitutive equations with Ting's model. As the relaxation modulus is a decaying function, it can be well described by rheological models such as a power-law rheology (PLR) model as used in this study ${ }^{84}$ :

$$
E(t)=E_{\infty}+\frac{E_{0}-E_{\infty}}{1+\left(t / t^{\prime}\right)^{\alpha}}
$$

Equation 4

where $E_{0}$ is the instantaneous modulus, $E_{\infty}$ the infinite modulus, $t^{\prime}$ is a small time offset (equal to $5^{*} 10^{-5} \mathrm{~s}$ in this study), and $\alpha$ is the power-law exponent (where $\alpha=0$ means a solid-like behavior and $\alpha=1$ a fluid-like behaviour). The modified PLR can be viewed as an infinite number of spring-and-dashpot combinations in parallel with removed zero time singularity, leading to a continuous relaxation spectrum and power-law decay ${ }^{84,106,107}$.

ii) Stretch exponential fit: AFM stress-relaxation measurements were performed by indenting and leaving the indenter to dwell in the film for $10 \mathrm{~s}$ while the z-piezo drive is paused and cantilever deflection i.e. force is monitored over time. The relaxation curves were fitted with a stretched exponential function of the form:

$$
F=F_{E}+F_{V} * \exp \left[-(t / \tau)^{\beta}\right]
$$

Equation 5

, where $F_{E}$ represents stored (elastic) stresses, $F_{V}$ are dissipated (viscous) stresses, $T$ is the relaxation time, and $\beta$ is a fractional power-law exponent $(0<\beta \leq 1)$ used for 'stretching'. A stretched exponential relaxation is commonly interpreted as a 'global relaxation' of a system that contains many independently relaxing species of which each decays exponentially in time with a 
specific relaxation rate ${ }^{91}$. A stretching exponent of $\beta=1$ results in simple exponential decay whereas smaller values of $\beta$ suggest non-exponential relaxation processes that show a 'fat tail' at long times, which originates from a continuous sum of exponential decays ${ }^{91,108}$. The stretching exponent $\beta$ is considered to be related to the logarithmic full width at half maximum (FWHM) of the relaxation time probability distribution $\mathrm{P}\left(\tau / \tau^{*}, \beta\right)$, where $\tau^{*}$ denotes a characteristic relaxation time. A physical interpretation of $\beta$ is that it represents a measure of the intrinsic small relaxation-rate cutoff of $\mathrm{P}\left(\tau / \tau^{*}, \beta\right)^{91}$. The stretched exponential function is commonly used to describe the relaxation behavior of homogeneous glasses as well as of linear viscoelastic media ${ }^{108-110}$. Non-linear curve fitting was performed with the software Origin (Originlab, USA) using the Levenberg-Marquardt algorithm. For each film 17 curves were fitted, each with a peak load $90 \mathrm{nN}$ as in indentation force-volume maps. Peak loads of $90 \mathrm{nN}$ force resulted in average indentation depths of $43 \pm 5 \mathrm{~nm}$ for drop-cast films and $58 \pm 8 \mathrm{~nm}$ for coagulation films. During the $10 \mathrm{~s}$ dwells the spherical indenter crept about $10 \mathrm{~nm}$ into the films due to unavoidable elastic stresses in the cantilever, resulting in a change of indentation area. However, this change in area was found to be generally smaller than the uncertainty of the measurements and for the sake of simplicity the area was considered constant for the stretch-exponential analysis ${ }^{111}$.

\section{Acknowledgements}

We thank the Aquarium in Ålesund 'Atlanterhavsparken' and in particular the curator Rune Veiseth and Trond Østrem for providing hagfish. We also thank Møreforsking for the possibility to use their facilities and for supervising the sampling of the hagfish according to the ethical guidelines (FOTS ID 6912). We thank Joe Berry and Srinivas Mettu for their helpful inputs regarding AFM measurements. We also thank Yuri Efremov for his extensive help regarding fitting and interpretation of his MATLAB code using Ting's model and for his comments on our manuscript. We acknowledge Sebastian Schade for his help regarding indenter choice.

\section{Competing Interests}

The authors declare no competing or financial interests.

\section{Author contributions}

Conceptualization: L.J.B., A.S-F., R.R.D., P.F.

Methodology: L.J.B.; A.S-F.

Formal analysis: L.J.B., M.W., A.S-F.

Investigation: L.J.B., M.W., A.S-F., M.B.

Data curation: L.J.B., M.W., A.S-F.

Writing - original draft: L.J.B.

Rewriting - review \& editing: L.J.B., A.S-F., R.R.D., R.M., E.J.W., P.F.

Visualization: L.J.B.

Supervision: P.F., E.J.W., R.R.D. R.M;

Project administration: P.F., E.J.W.

Funding acquisition: P.F., E.J.W.

\section{Funding}


This work was supported by ETH Research Grant ETH-19 14-1. The travel expenses for M.W. were partly funded by the Walter Hochstrasser Foundation. 


\section{References}

(1) Herrmann, H.; Bär, H.; Kreplak, L.; Strelkov, S. V.; Aebi, U. Intermediate Filaments: From Cell Architecture to Nanomechanics. Nat. Rev. Mol. Cell Biol. 2007, 8, 562-573.

(2) Fuchs, E.; Cleveland, D. W. A Structural Scaffolding of Intermediate Filaments in Health and Disease. Science 1998, 279, 514-519.

(3) Magin, T. M.; Hesse, M.; Schröder, R. Novel Insights into Intermediate-Filament Function from Studies of Transgenic and Knockout Mice. Protoplasma 2000, 211, 140-150.

(4) Qin, Z.; Buehler, M. J.; Kreplak, L. A Multi-Scale Approach to Understand the Mechanobiology of Intermediate Filaments. J. Biomech. 2010, 43, 15-22.

(5) Ramms, L.; Fabris, G.; Windoffer, R.; Schwarz, N.; Springer, R.; Zhou, C.; Lazar, J.; Stiefel, S.; Hersch, N.; Schnakenberg, U.; et al. Keratins as the Main Component for the Mechanical Integrity of Keratinocytes. Proc. Natl. Acad. Sci. U. S. A. 2013, 110, 1851318518.

(6) Seltmann, K.; Fritsch, A. W.; Käs, J. A.; Magin, T. M. Keratins Significantly Contribute to Cell Stiffness and Impact Invasive Behavior. Proc. Natl. Acad. Sci. U. S. A. 2013, 110, 18507-18512.

(7) Strelkov, S. V.; Herrmann, H.; Aebi, U. Molecular Architecture of Intermediate Filaments. Bioessays 2003, 25, 243-251.

(8) Block, J.; Schroeder, V.; Pawelzyk, P.; Willenbacher, N.; Köster, S. Physical Properties of Cytoplasmic Intermediate Filaments. Biochimica et Biophysica Acta (BBA) - Molecular Cell Research 2015, 1853, 3053-3064.

(9) Kreplak, L.; Fudge, D. Biomechanical Properties of Intermediate Filaments: From Tissues to Single Filaments and Back. Bioessays 2007, 29, 26-35.

(10) Köster, S.; Weitz, D. A.; Goldman, R. D.; Aebi, U.; Herrmann, H. Intermediate Filament Mechanics in Vitro and in the Cell: From Coiled Coils to Filaments, Fibers and Networks. Curr. Opin. Cell Biol. 2015, 32, 82-91.

(11) Mücke, N.; Kreplak, L.; Kirmse, R.; Wedig, T.; Herrmann, H.; Aebi, U.; Langowski, J. Assessing the Flexibility of Intermediate Filaments by Atomic Force Microscopy. J. Mol. Biol. 2004, 335, 1241-1250.

(12) Kreplak, L.; Herrmann, H.; Aebi, U. Tensile Properties of Single Desmin Intermediate Filaments. Biophys. J. 2008, 94, 2790-2799.

(13) Guzmán, C.; Jeney, S.; Kreplak, L.; Kasas, S.; Kulik, A. J.; Aebi, U.; Forró, L. Exploring the Mechanical Properties of Single Vimentin Intermediate Filaments by Atomic Force Microscopy. J. Mol. Biol. 2006, 360, 623-630.

(14) Nöding, B.; Köster, S. Intermediate Filaments in Small Configuration Spaces. Phys. Rev. Lett. 2012, 108, 088101.

(15) Kreplak, L.; Bär, H.; Leterrier, J. F.; Herrmann, H.; Aebi, U. Exploring the Mechanical Behavior of Single Intermediate Filaments. J. Mol. Biol. 2005, 354, 569-577.

(16) Block, J.; Witt, H.; Candelli, A.; Peterman, E. J. G.; Wuite, G. J. L.; Janshoff, A.; Köster, S. Nonlinear Loading-Rate-Dependent Force Response of Individual Vimentin Intermediate Filaments to Applied Strain. Phys. Rev. Lett. 2017, 118, 048101.

(17) Downing, S. W.; Spitzer, R. H.; Koch, E. A.; Salo, W. L. The Hagfish Slime Gland Thread Cell. I. A Unique Cellular System for the Study of Intermediate Filaments and Intermediate Filament-Microtubule Interactions. J. Cell Biol. 1984, 98, 653-669.

(18) Spitzer, R. H.; Koch, E. A.; Downing, S. W. Maturation of Hagfish Gland Thread Cells: Composition and Characterization of Intermediate Filament Polypeptides. Cell Motil. Cytoskeleton 1988, 11, 31-45.

(19) Koch, E. A.; Spitzer, R. H.; Pithawalla, R. B.; Castillos, F. A., 3rd; Parry, D. A. Hagfish Biopolymer: A Type I/type II Homologue of Epidermal Keratin Intermediate Filaments. Int. J. Biol. Macromol. 1995, 17, 283-292. 
(20) Spitzer, R. H.; Downing, S. W.; Koch, E. A.; Salo, W. L.; Saidel, L. J. Hagfish Slime Gland Thread Cells. II. Isolation and Characterization of Intermediate Filament Components Associated with the Thread. J. Cell Biol. 1984, 98, 670-677.

(21) Koch, E. A.; Spitzer, R. H.; Pithawalla, R. B.; Parry, D. A. An Unusual Intermediate Filament Subunit from the Cytoskeletal Biopolymer Released Extracellularly into Seawater by the Primitive Hagfish (Eptatretus Stouti). J. Cell Sci. 1994, 107, 3133-3144.

(22) Parker, T. J.; Jeffery Parker, T.; Haswell, W. A. A Text-Book of Zoology; Macmillan, 1910.

(23) Jørgensen, J. M.; Lomholt, J. P.; Weber, R. E.; Malte, H. The Biology of Hagfishes; Springer Science \& Business Media, 2012.

(24) Fudge, D. S.; Gardner, K. H.; Forsyth, V. T.; Riekel, C.; Gosline, J. M. The Mechanical Properties of Hydrated Intermediate Filaments: Insights from Hagfish Slime Threads. Biophys. J. 2003, 85, 2015-2027.

(25) Fudge, D. S.; Levy, N.; Chiu, S.; Gosline, J. M. Composition, Morphology and Mechanics of Hagfish Slime. J. Exp. Biol. 2005, 208, 4613-4625.

(26) Ewoldt, R. H.; Winegard, T. M.; Fudge, D. S. Non-Linear Viscoelasticity of Hagfish Slime. Int. J. Non Linear Mech. 2011, 46, 627-636.

(27) Böni, L.; Fischer, P.; Böcker, L.; Kuster, S.; Rühs, P. A. Hagfish Slime and Mucin Flow Properties and Their Implications for Defense. Sci. Rep. 2016, 6, 30371.

(28) Fernholm, B. Thread Cells from the Slime Glands of Hagfish (Myxinidae). Acta Zool. 1981, 62, 137-145.

(29) Downing, S. W.; Spitzer, R. H.; Salo, W. L.; Downing, J. S.; Saidel, L. J.; Koch, E. A. Threads in the Hagfish Slime Gland Thread Cells: Organization, Biochemical Features, and Length. Science 1981, 212, 326-328.

(30) Fudge, D. S.; Schorno, S.; Ferraro, S. Physiology, Biomechanics, and Biomimetics of Hagfish Slime. Annu. Rev. Biochem. 2015, 84, 947-967.

(31) Schaffeld, M.; Schultess, J. Genes Coding for Intermediate Filament Proteins Closely Related to the Hagfish "thread Keratins (TK)" $\alpha$ and y Also Exist in Lamprey, Teleosts and Amphibians. Exp. Cell Res. 2006, 312, 1447-1462.

(32) Fu, J.; Guerette, P. A.; Pavesi, A.; Horbelt, N.; Lim, C. T.; Harrington, M. J.; Miserez, A. Artificial Hagfish Protein Fibers with Ultra-High and Tunable Stiffness. Nanoscale 2017, 9, 12908-12915.

(33) Fu, J.; Guerette, P. A.; Miserez, A. Self-Assembly of Recombinant Hagfish Thread Keratins Amenable to a Strain-Induced $\alpha$-Helix to $\beta$-Sheet Transition. Biomacromolecules 2015, 16, 2327-2339.

(34) Downing, S. W.; Salo, W. L.; Spitzer, R. H.; Koch, E. A. The Hagfish Slime Gland: A Model System for Studying the Biology of Mucus. Science 1981, 214, 1143-1145.

(35) Fudge, D. S.; Gosline, J. M. Molecular Design of the Alpha-Keratin Composite: Insights from a Matrix-Free Model, Hagfish Slime Threads. Proc. Biol. Sci. 2004, 271, 291-299.

(36) Negishi, A.; Armstrong, C. L.; Kreplak, L.; Rheinstadter, M. C.; Lim, L.-T.; Gillis, T. E.; Fudge, D. S. The Production of Fibers and Films from Solubilized Hagfish Slime Thread Proteins. Biomacromolecules 2012, 13, 3475-3482.

(37) Feughelman, M. A Two-Phase Structure for Keratin Fibers. Text. Res. J. 1959, 29, 223228.

(38) Charrier, E. E.; Janmey, P. A. Mechanical Properties of Intermediate Filament Proteins. Methods Enzymol. 2016, 568, 35-57.

(39) Fraser, R. D.; Macrae, T. P. Molecular Structure and Mechanical Properties of Keratins. Symp. Soc. Exp. Biol. 1980, 34, 211-246.

(40) Fudge, D. S.; Winegard, T.; Ewoldt, R. H.; Beriault, D.; Szewciw, L.; McKinley, G. H. From Ultra-Soft Slime to Hard -Keratins: The Many Lives of Intermediate Filaments. Integr. Comp. Biol. 2009, 49, 32-39.

(41) Fey, E. G. Epithelial Cytoskeletal Framework and Nuclear Matrix-Intermediate Filament 
Scaffold: Three-Dimensional Organization and Protein Composition. J. Cell Biol. 1984, 98, 1973-1984.

(42) Reichl, S.; Borrelli, M.; Geerling, G. Keratin Films for Ocular Surface Reconstruction. Biomaterials 2011, 32, 3375-3386.

(43) Xiong, R.; Grant, A. M.; Ma, R.; Zhang, S.; Tsukruk, V. V. Naturally-Derived Biopolymer Nanocomposites: Interfacial Design, Properties and Emerging Applications. Mater. Sci. Eng. R Rep. 2018, 125, 1-41.

(44) Gladman, A. S.; Matsumoto, E. A.; Nuzzo, R. G.; Mahadevan, L.; Lewis, J. A. Biomimetic 4D Printing. Nat. Mater. 2016, 15, 413-418.

(45) Marelli, B.; Brenckle, M. A.; Kaplan, D. L.; Omenetto, F. G. Silk Fibroin as Edible Coating for Perishable Food Preservation. Sci. Rep. 2016, 6, 25263.

(46) Mapp, K. US Navy Synthetically Recreates Biomaterial To Assist Military Personnel https://www.navy.mil/submit/display.asp?story_id=98521.

(47) Desai, A.; Mitchison, T. J. Microtubule Polymerization Dynamics. Annu. Rev. Cell Dev. Biol. 1997, 13, 83-117.

(48) Winegard, T.; Herr, J.; Mena, C.; Lee, B.; Dinov, I.; Bird, D.; Bernards, M., Jr; Hobel, S.; Van Valkenburgh, B.; Toga, A.; et al. Coiling and Maturation of a High-Performance Fibre in Hagfish Slime Gland Thread Cells. Nat. Commun. 2014, 5, 3534.

(49) Salo, W. L.; Downing, S. W.; Lidinsky, W. A.; Gallagher, W. H.; Spitzer, R. H.; Koch, E. A. Fractionation of Hagfish Slime Gland Secretions: Partial Characterization of the Mucous Vesicle Fraction. Prep. Biochem. 1983, 13, 103-135.

(50) Lehtonen, A.; Kärkkäinen, J.; Haahti, E. Carbohydrate Components in the Epithelial Mucin of Hagfish, Myxine Glutinosa. Acta Chem. Scand. 1966, 20, 1456-1462.

(51) Powell, D. A.; Turula, V.; Dehaseth, J. A.; Vanhalbeek, H.; Meyer, B. Sulfate Detection in Glycoprotein-Derived Oligosaccharides by Artificial Neural Network Analysis FourierTransform Infrared Spectra. Anal. Biochem. 1994, 220, 20-27.

(52) Lewis, A. T.; Jones, K.; Lewis, K. E.; Jones, S.; Lewis, P. D. Detection of Lewis Antigen Structural Change by FTIR Spectroscopy. Carbohydr. Polym. 2013, 92, 1294-1301.

(53) Nikonenko, N. A.; Bushnak, I. A.; Keddie, J. L. Spectroscopic Ellipsometry of Mucin Layers on an Amphiphilic Diblock Copolymer Surface. Appl. Spectrosc. 2009, 63, 889-898.

(54) Lewis, S. P.; Lewis, A. T.; Lewis, P. D. Prediction of Glycoprotein Secondary Structure Using ATR-FTIR. Vib. Spectrosc. 2013, 69, 21-29.

(55) Ahn, J.; Crouzier, T.; Ribbeck, K.; Rubner, M. F.; Cohen, R. E. Tuning the Properties of Mucin via Layer-by-Layer Assembly. Biomacromolecules 2015, 16, 228-235.

(56) Bavington, C. D.; Lever, R.; Mulloy, B.; Grundy, M. M.; Page, C. P.; Richardson, N. V.; McKenzie, J. D. Anti-Adhesive Glycoproteins in Echinoderm Mucus Secretions. Comp. Biochem. Physiol. B Biochem. Mol. Biol. 2004, 139, 607-617.

(57) Lalonde, S. V.; Dafoe, L. T.; Pemberton, S. G.; Gingras, M. K.; Konhauser, K. O. Investigating the Geochemical Impact of Burrowing Animals: Proton and Cadmium Adsorption onto the Mucus Lining of Terebellid Polychaete Worms. Chem. Geol. 2010, 271, 44-51.

(58) Hu, X.; Kaplan, D.; Cebe, P. Determining Beta-Sheet Crystallinity in Fibrous Proteins by Thermal Analysis and Infrared Spectroscopy. Macromolecules 2006, 39, 6161-6170.

(59) Mezzenga, R.; Fischer, P. The Self-Assembly, Aggregation and Phase Transitions of Food Protein Systems in One, Two and Three Dimensions. Rep. Prog. Phys. 2013, 76, 046601.

(60) Schwegman, J. J.; Carpenter, J. F.; Nail, S. L. Infrared Microscopy for in Situ Measurement of Protein Secondary Structure during Freezing and Freeze-Drying. J. Pharm. Sci. 2007, 96, 179-195.

(61) Herrmann, H.; Aebi, U. Intermediate Filaments: Molecular Structure, Assembly Mechanism, and Integration into Functionally Distinct Intracellular Scaffolds. Annu. Rev. Biochem. 2004, 73, 749-789. 
(62) Fraser, R. D. B.; MacRae, T. P.; Parry, D. A. D.; Suzuki, E. The Structure of $\beta$-Keratin. Polymer 1969, 10, 810-826.

(63) Kornreich, M.; Avinery, R.; Malka-Gibor, E.; Laser-Azogui, A.; Beck, R. Order and Disorder in Intermediate Filament Proteins. FEBS Lett. 2015, 589, 2464-2476.

(64) Beck, R.; Deek, J.; Jones, J. B.; Safinya, C. R. Gel-Expanded to Gel-Condensed Transition in Neurofilament Networks Revealed by Direct Force Measurements. Nat. Mater. 2010, 9, 40-46.

(65) Beck, R.; Deek, J.; Choi, M. C.; Ikawa, T.; Watanabe, O.; Frey, E.; Pincus, P.; Safinya, C. R. Unconventional Salt Trend from Soft to Stiff in Single Neurofilament Biopolymers. Langmuir 2010, 26, 18595-18599.

(66) Wen, Q.; Janmey, P. A. Polymer Physics of the Cytoskeleton. Curr. Opin. Solid State Mater. Sci. 2011, 15, 177-182.

(67) Janmey, P. A.; Slochower, D. R.; Wang, Y.-H.; Wen, Q.; Cēbers, A. Polyelectrolyte Properties of Filamentous Biopolymers and Their Consequences in Biological Fluids. Soft Matter 2014, 10, 1439-1449.

(68) Kumar, S.; Yin, X.; Trapp, B. D.; Hoh, J. H.; Paulaitis, M. E. Relating Interactions between Neurofilaments to the Structure of Axonal Neurofilament Distributions through Polymer Brush Models. Biophys. J. 2002, 82, 2360-2372.

(69) Zhulina, E. B.; Leermakers, F. A. M. The Polymer Brush Model of Neurofilament Projections: Effect of Protein Composition. Biophys. J. 2010, 98, 462-469.

(70) Koch, E. Structural Forms and Possible Roles of Aligned Cytoskeletal Biopolymers in Hagfish (slime Eel) mucus*1. J. Struct. Biol. 1991, 106, 205-210.

(71) Böni, L. J.; Zurflüh, R.; Baumgartner, M. E.; Windhab, E. J.; Fischer, P.; Kuster, S.; Rühs, P. A. Effect of Ionic Strength and Seawater Cations on Hagfish Slime Formation. Sci. Rep. 2018, 8, 9867.

(72) Brown, H. G.; Hoh, J. H. Entropic Exclusion by Neurofilament Sidearms: A Mechanism for Maintaining Interfilament Spacing. Biochemistry 1997, 36, 15035-15040.

(73) Fudge, D. S.; Hillis, S.; Levy, N.; Gosline, J. M. Hagfish Slime Threads as a Biomimetic Model for High Performance Protein Fibres. Bioinspir. Biomim. 2010, 5, 035002.

(74) Parbhu, A. N.; Bryson, W. G.; Lal, R. Disulfide Bonds in the Outer Layer of Keratin Fibers Confer Higher Mechanical Rigidity: Correlative Nano-Indentation and Elasticity Measurement with an AFM. Biochemistry 1999, 38, 11755-11761.

(75) Bhushan, B.; Chen, N. AFM Studies of Environmental Effects on Nanomechanical Properties and Cellular Structure of Human Hair. Ultramicroscopy 2006, 106, 755-764.

(76) Farran, L.; Roland Ennos, A.; Eichhorn, S. J. Microindentation and Nanoindentation of Human Fingernails at Varying Relative Humidity. J. Mater. Res. 2009, 24, 980-984.

(77) Lin, Y.-C.; Yao, N. Y.; Broedersz, C. P.; Herrmann, H.; Mackintosh, F. C.; Weitz, D. A. Origins of Elasticity in Intermediate Filament Networks. Phys. Rev. Lett. 2010, 104, 058101.

(78) Zhao, X. Multi-Scale Multi-Mechanism Design of Tough Hydrogels: Building Dissipation into Stretchy Networks. Soft Matter 2014, 10, 672-687.

(79) Rebelo, L. M.; de Sousa, J. S.; Mendes Filho, J.; Radmacher, M. Comparison of the Viscoelastic Properties of Cells from Different Kidney Cancer Phenotypes Measured with Atomic Force Microscopy. Nanotechnology 2013, 24, 055102.

(80) Cappella, B. Mechanical Properties of Polymers Measured through AFM Force-Distance Curves; Springer Laboratory; Springer International Publishing: Cham, 2016.

(81) Schopferer, M.; Bär, H.; Hochstein, B.; Sharma, S.; Mücke, N.; Herrmann, H.; Willenbacher, N. Desmin and Vimentin Intermediate Filament Networks: Their Viscoelastic Properties Investigated by Mechanical Rheometry. J. Mol. Biol. 2009, 388, 133-143.

(82) Uhlig, M. R.; Magerle, R. Unraveling Capillary Interaction and Viscoelastic Response in Atomic Force Microscopy of Hydrated Collagen Fibrils. Nanoscale 2017, 9, 1244-1256. 
(83) Yuan, Y.; Verma, R. Measuring Microelastic Properties of Stratum Corneum. Colloids Surf. B Biointerfaces 2006, 48, 6-12.

(84) Efremov, Y. M.; Wang, W.-H.; Hardy, S. D.; Geahlen, R. L.; Raman, A. Measuring Nanoscale Viscoelastic Parameters of Cells Directly from AFM Force-Displacement Curves. Sci. Rep. 2017, 7, 1541.

(85) Efremov, Y. M.; Bagrov, D. V.; Kirpichnikov, M. P.; Shaitan, K. V. Application of the Johnson-Kendall-Roberts Model in AFM-Based Mechanical Measurements on Cells and Gel. Colloids Surf. B Biointerfaces 2015, 134, 131-139.

(86) Chen, J. F.; Xu, K. J.; Tang, L. Q.; Liu, Z. J.; Zhou, L. C. Study on the Optimal Loading Rates in the Measurement of Viscoelastic Properties of Hydrogels by Conical Indentation. Mech. Mater. 2018, 119, 42-48.

(87) Attard, P. Measurement and Interpretation of Elastic and Viscoelastic Properties with the Atomic Force Microscope. J. Phys. Condens. Matter 2007, 19, 473201.

(88) Gillies, G.; Prestidge, C. A.; Attard, P. An AFM Study of the Deformation and Nanorheology of Cross-Linked PDMS Droplets. Langmuir 2002, 18, 1674-1679.

(89) Ting, T. C. T. The Contact Stresses Between a Rigid Indenter and a Viscoelastic HalfSpace. J. Appl. Mech. 1966, 33, 845.

(90) Nawaz, S.; Sánchez, P.; Bodensiek, K.; Li, S.; Simons, M.; Schaap, I. A. T. Cell ViscoElasticity Measured with AFM and Optical Trapping at Sub-Micrometer Deformations. PLoS One 2012, 7, e45297.

(91) Johnston, D. C. Stretched Exponential Relaxation Arising from a Continuous Sum of Exponential Decays. Phys. Rev. B: Condens. Matter Mater. Phys. 2006, 74.

(92) Meng, F.; Pritchard, R. H.; Terentjev, E. M. Stress Relaxation, Dynamics, and Plasticity of Transient Polymer Networks. Macromolecules 2016, 49, 2843-2852.

(93) Pawelzyk, P.; Mücke, N.; Herrmann, H.; Willenbacher, N. Attractive Interactions among Intermediate Filaments Determine Network Mechanics in Vitro. PLoS One 2014, 9, e93194.

(94) Kroy, K.; Glaser, J. The Glassy Wormlike Chain. New J. Phys. 2007, 9, 416-416.

(95) Perticaroli, S.; Nickels, J. D.; Ehlers, G.; O’Neill, H.; Zhang, Q.; Sokolov, A. P. Secondary Structure and Rigidity in Model Proteins. Soft Matter 2013, 9, 9548.

(96) Perticaroli, S.; Nickels, J. D.; Ehlers, G.; Sokolov, A. P. Rigidity, Secondary Structure, and the Universality of the Boson Peak in Proteins. Biophys. J. 2014, 106, 2667-2674.

(97) De Rosa, C.; Auriemma, F. Crystals and Crystallinity in Polymers: Diffraction Analysis of Ordered and Disordered Crystals; John Wiley \& Sons, 2013.

(98) Zugenmaier, P. X-Ray Analysis of Partial Crystalline Fibre Structure. In Handbook of Textile Fibre Structure; 2009; pp. 46-120.

(99) Hermans, P. H.; Platzek, P. Beiträge Zur Kenntnis Des Deformationsmechanismus Und Der Feinstruktur Der Hydratzellulose. Kolloid-Zeitschrift 1939, 88, 68-72.

(100) Böni, L. J.; Zurflüh, R.; Widmer, M.; Fischer, P.; Windhab, E. J.; Rühs, P. A.; Kuster, S. Hagfish Slime Exudate Stabilization and Its Effect on Slime Formation and Functionality. Biol. Open 2017, 6, 1115-1122.

(101) Zou, Y.; Li, Y.; Hao, W.; Hu, X.; Ma, G. Parallel $\beta$-Sheet Fibril and Antiparallel $\beta$-Sheet Oligomer: New Insights into Amyloid Formation of Hen Egg White Lysozyme under Heat and Acidic Condition from FTIR Spectroscopy. J. Phys. Chem. B 2013, 117, 4003-4013.

(102) Dimitriadis, E. K.; Horkay, F.; Maresca, J.; Kachar, B.; Chadwick, R. S. Determination of Elastic Moduli of Thin Layers of Soft Material Using the Atomic Force Microscope. Biophys. J. 2002, 82, 2798-2810.

(103) Niu, T.; Cao, G. Finite Size Effect Does Not Depend on the Loading History in Soft Matter Indentation. J. Phys. D Appl. Phys. 2014, 47, 385303.

(104) Fischer-Cripps, A. C. Nanoindentation; Springer New York, 2011.

(105) Lee, E. H.; Radok, J. R. M. The Contact Problem for Viscoelastic Bodies. J. Appl. Mech. 
1960, 27, 438.

(106) Bagley, R. L. Power Law and Fractional Calculus Model of Viscoelasticity. AIAA Journal 1989, 27, 1412-1417.

(107) Sharpe, W. N. Springer Handbook of Experimental Solid Mechanics; Springer Science \& Business Media, 2008.

(108) Mauro, J. C.; Mauro, Y. Z. On the Prony Series Representation of Stretched Exponential Relaxation. Physica A: Statistical Mechanics and its Applications 2018, 506, 75-87.

(109) Berry, G. C.; Plazek, D. J. On the Use of Stretched-Exponential Functions for Both Linear Viscoelastic Creep and Stress Relaxation. Rheol. Acta 1997, 36, 320-329.

(110) Zanzotto, L.; Stastna, J. Dynamic Master Curves from the Stretched Exponential Relaxation Modulus. J. Polym. Sci. B Polym. Phys. 1997, 35, 1225-1232.

(111) Uzüm, C.; Hellwig, J.; Madaboosi, N.; Volodkin, D.; von Klitzing, R. Growth Behaviour and Mechanical Properties of PLL/HA Multilayer Films Studied by AFM. Beilstein J. Nanotechnol. 2012, 3, 778-788. 


\section{Supplementary Information}

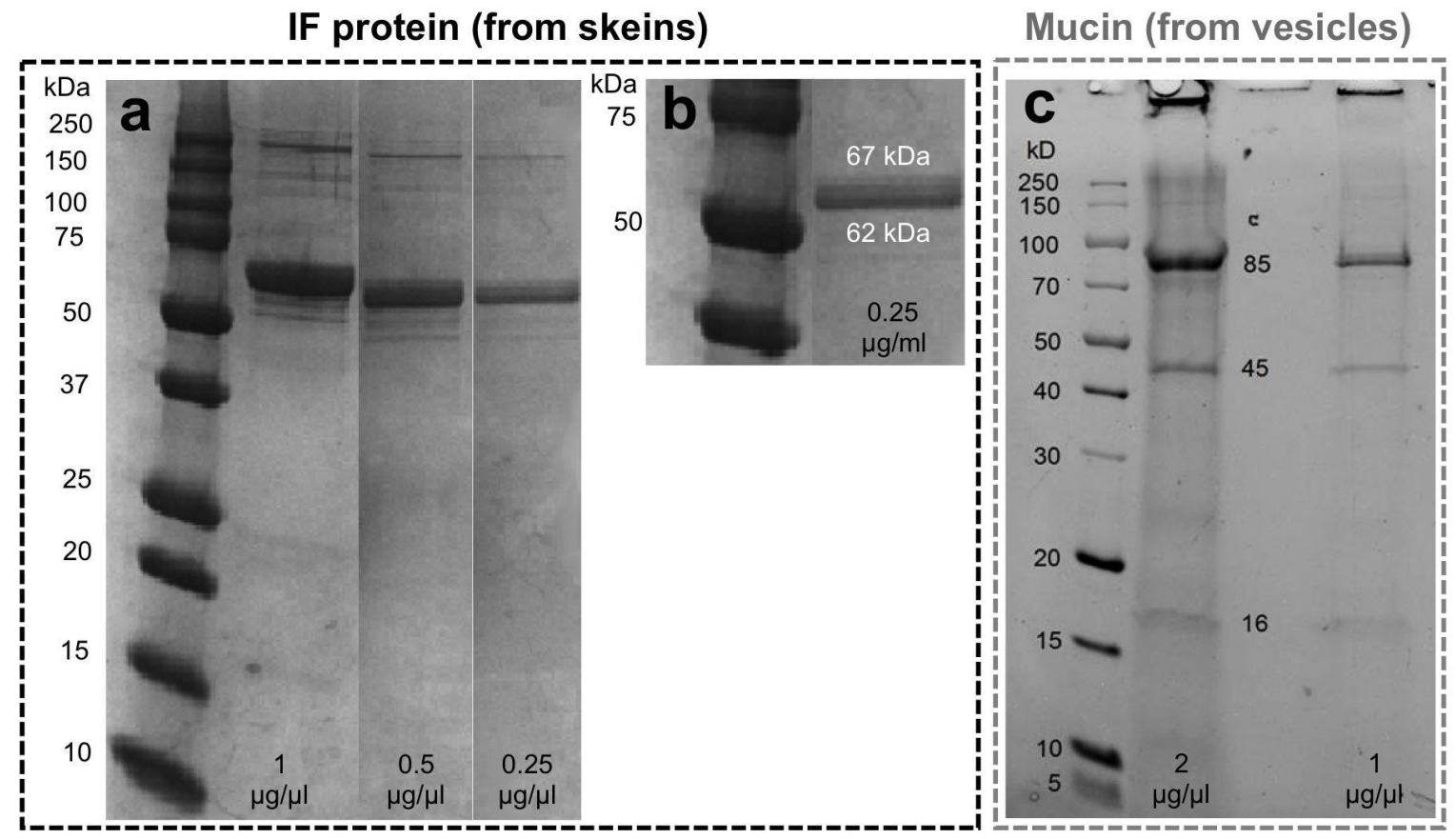

Figure S1: SDS-PAGE of freeze-dried hagfish skeins and mucin. (a) Skeins (hagfish fiber intermediate filament protein) at various concentrations consistently show a double band around 62 to 67 $\mathrm{kDa}$. (b) Magnification of double band from (a) at a sample concentration of $0.25 \mu \mathrm{g} / \mu \mathrm{l}$. (c) Dialyzed hagfish mucin

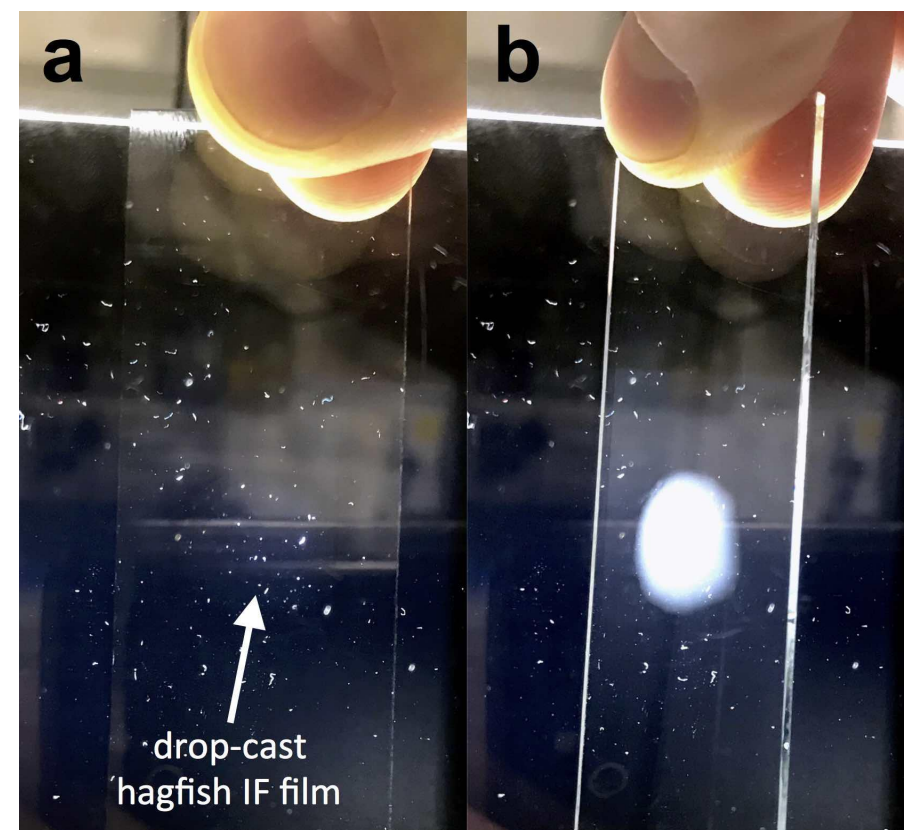

Figure S2: Birefringence of hagfish IF film drop-cast on glass. (a) Holding the glass slide parallel to the cross-polarizers does not result in birefringence. (b) When the glass slide is tilted birefringence is observed. This suggests that the $\beta$-sheets in the IF $\beta$-crystallites are parallel to the glass substrate. Therefore, when the film is held parallel to the cross-polarizers, the incident light is perpendicular to the $\beta$-sheets and thus no birefringence is observed. 

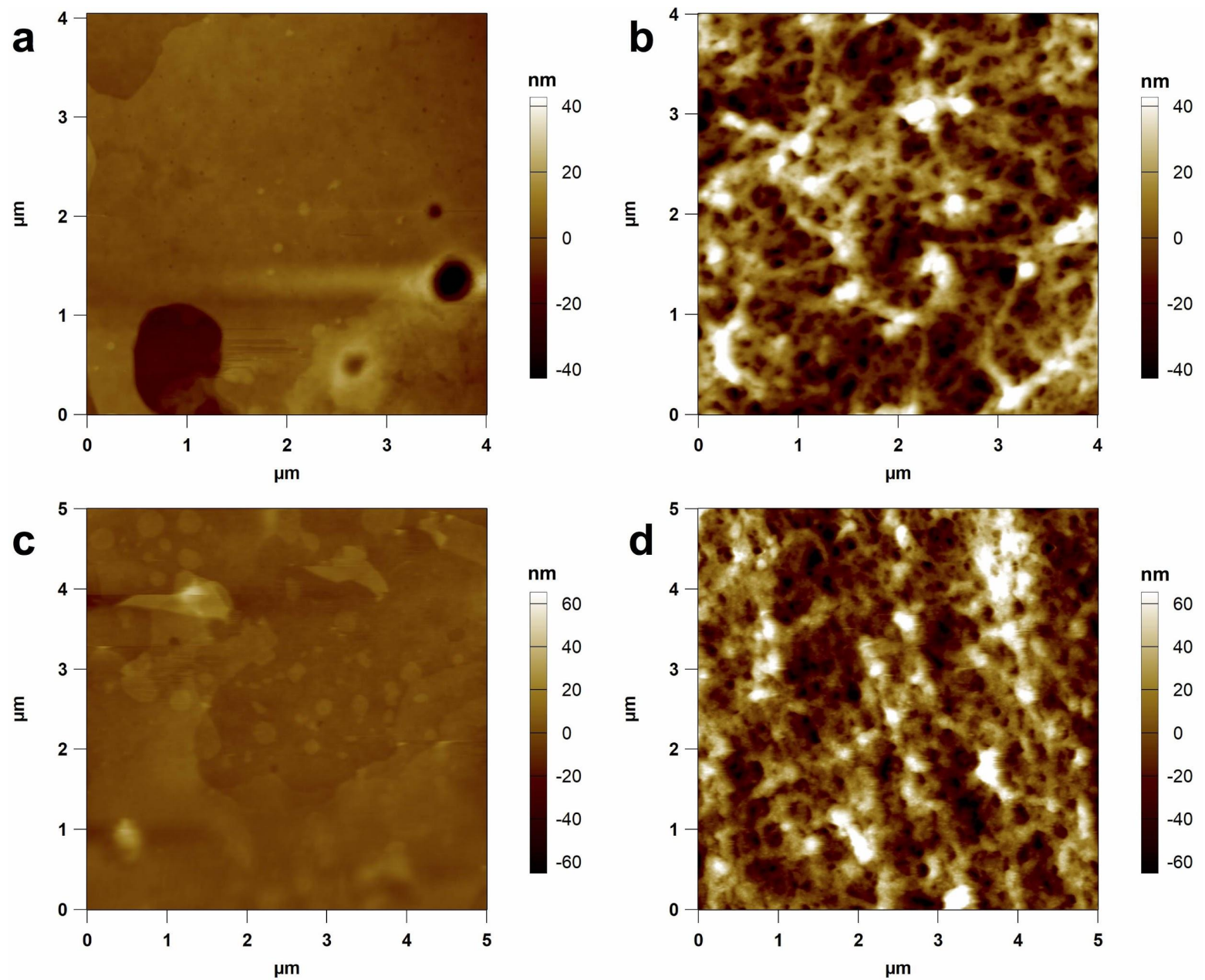

Figure S3: AFM images of a drop-cast film (a) and a coagulation film (b) in the dry state. AFM images of a drop-cast film (c) and a coagulation film (d) in liquid $(5.45 \mathrm{mM} \mathrm{NaCl}+0.1 \mathrm{mM} \mathrm{CaCl}$ ).
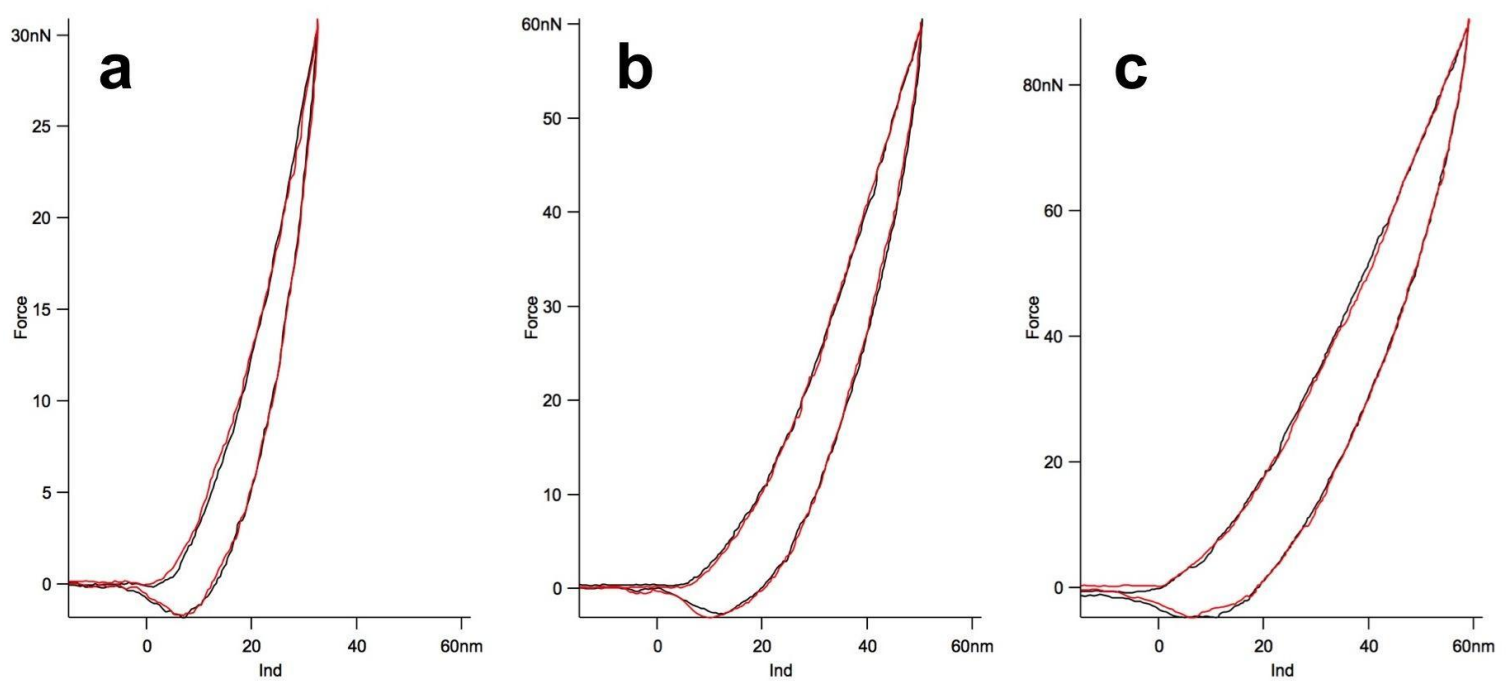

Figure S4: Repeated indentation on the same spot at increasing trigger force on a coagulation film. Red curve - 1st indentation, black curve 6th indentation for increasing trigger forces (a) $<$ (b) $<$ (c). The hysteresis loop does not change with repeated indentations on the same spot, showing that there is no permanent plastic deformation. 

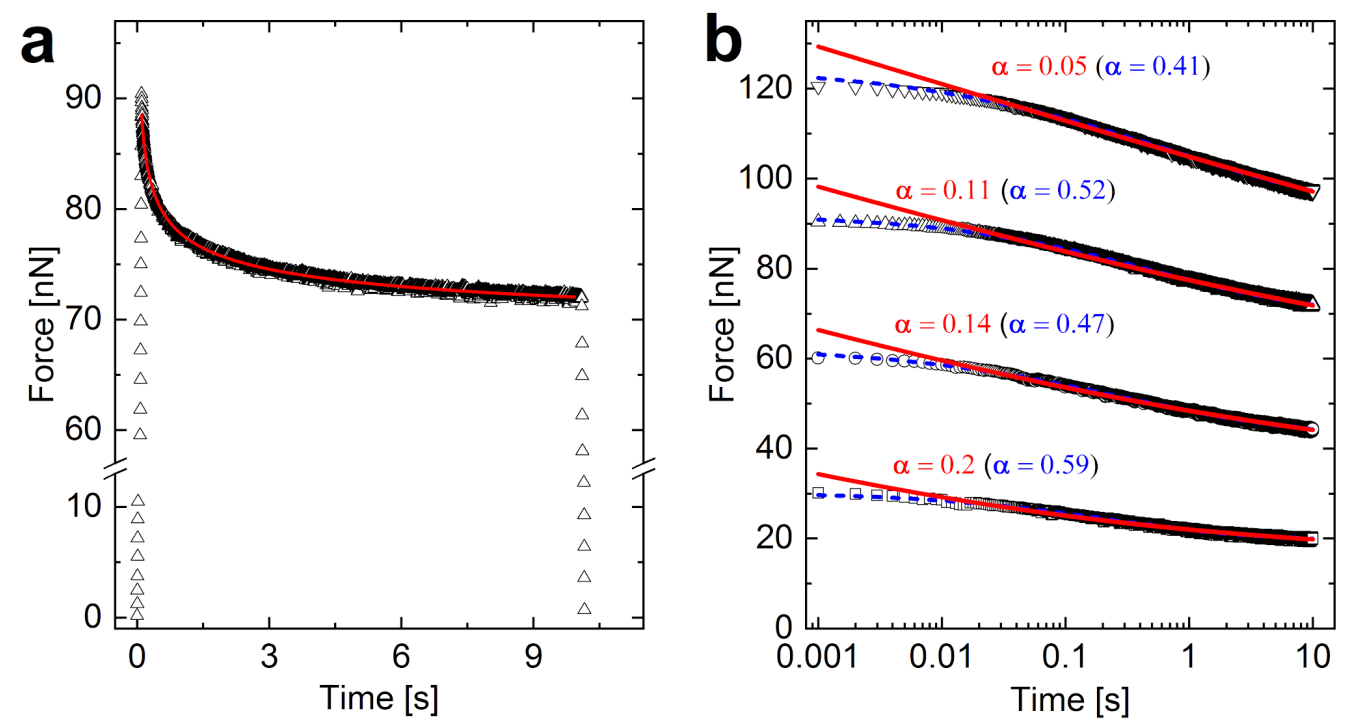

Figure S5: Power-law rheology (PLR) model fitted to AFM stress-relaxation measurements on a hydrated hagfish IF film (coagulation film). (a) Stress relaxation from an initial load of about $90 \mathrm{nN}$ with fitted PLR model. (b) Stress relaxation measurements performed at increasing initial peak load (strain) and the corresponding PLR fits. The red lines depict fits where $t^{\prime}$ was held constant at $5^{*} 10^{-5}$, similar to the PLR fits used for the F-D curves. The blue lines represent fits where t' was let run free for the fitting, which resulted in better fits but also in substantially higher alpha values (in brackets).
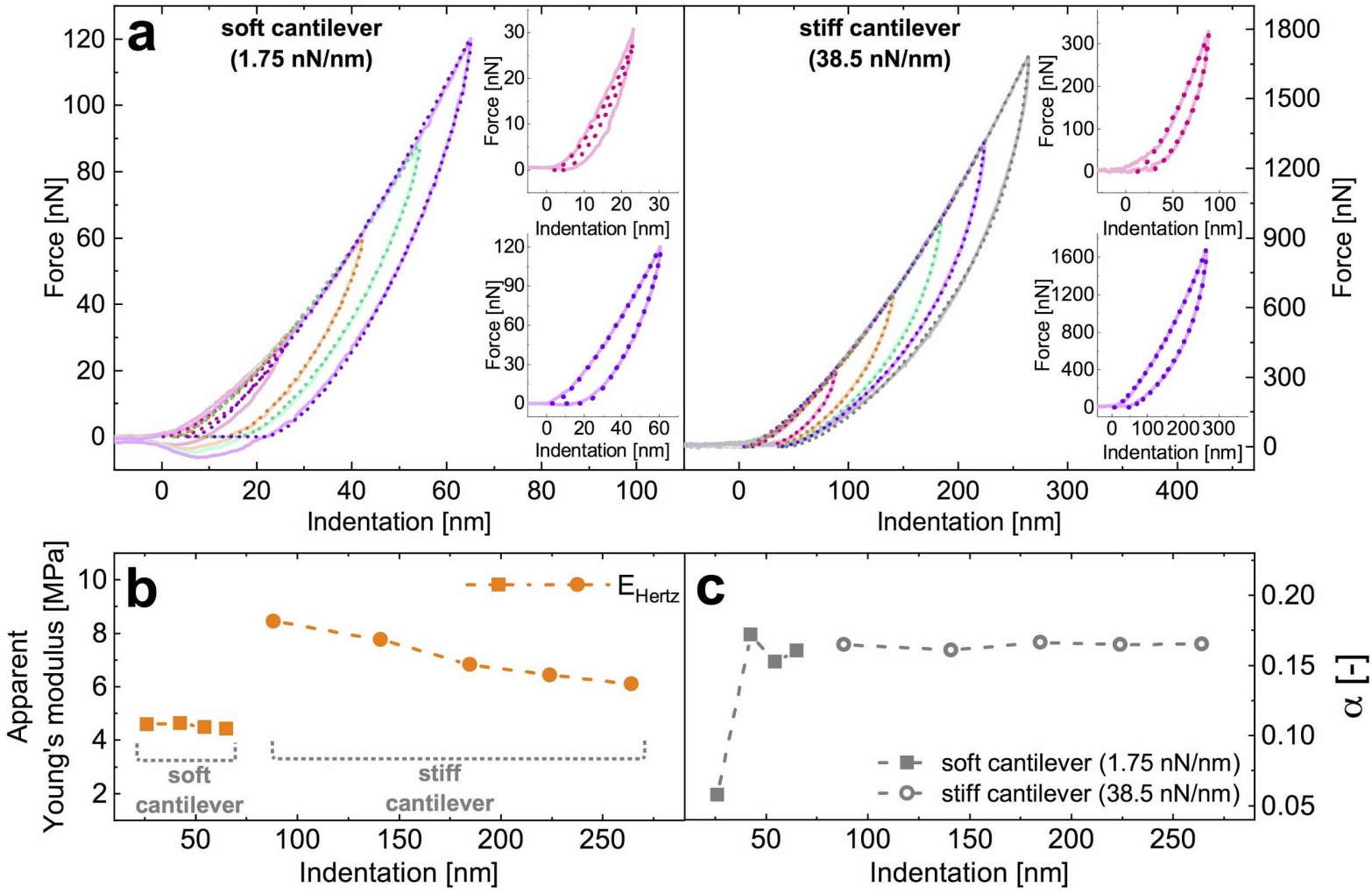

Figure S6: Effect of increasing indentation depth (strain) for two differently stiff cantilevers on the mechanical properties of hydrated coagulation film. (a) Force-distance curves (solid lines) performed at increasing peak forces on a hydrated coagulation film, overlaid with the PLR fit. The graph on the left shows curves obtained with a soft cantilever $(1.75 \mathrm{nN} / \mathrm{nm})$ and the graph on the right shows curves obtained using a stiff cantilever $(38.5 \mathrm{nN} / \mathrm{nm})$. Both cantilevers had a radius of $500 \mathrm{~nm}$. (b) Elastic modulus and (c) power-law exponent $a$ based on data shown in (a). 
Table S1: Root mean square (RMS) and average deviation (ADev) roughness of dry and hydrated hagfish IF films calculated for $1 \times 1 \mu \mathrm{m}$ and $2 \times 2 \mu \mathrm{m}$ window sizes.

\begin{tabular}{|c|c|c|c|c|c|c|c|c|}
\hline & \multicolumn{4}{|c|}{ Coagulation film } & \multicolumn{4}{|c|}{ Drop-cast film } \\
\hline & \multicolumn{2}{|c|}{ dry } & \multicolumn{2}{|c|}{ hydrated } & \multicolumn{2}{|c|}{ dry } & \multicolumn{2}{|c|}{ hydrated } \\
\hline & $\begin{array}{l}1 \times 1 \mu \mathrm{m} \\
\text { window } \\
(\mathrm{n}=25)\end{array}$ & $\begin{array}{l}2 \times 2 \mu \mathrm{m} \\
\text { window } \\
(\mathrm{n}=5)\end{array}$ & $\begin{array}{l}1 \times 1 \mu \mathrm{m} \\
\text { window } \\
(\mathrm{n}=25)\end{array}$ & $\begin{array}{l}2 \times 2 \mu m \\
\text { window d } \\
(n=25)\end{array}$ & $\begin{array}{l}1 \times 1 \mu \mathrm{m} \\
\text { window } \\
(\mathrm{n}=25)\end{array}$ & $\begin{array}{l}2 \times 2 \mu \mathrm{m} \\
\text { window } \\
(n=5)\end{array}$ & $\begin{array}{l}1 \times 1 \mu \mathrm{m} \\
\text { window } \\
(\mathrm{n}=5)\end{array}$ & $\begin{array}{l}2 \times 2 \mu \mathrm{m} \\
\text { window } \\
\mathrm{n}=5)\end{array}$ \\
\hline $\begin{array}{l}\text { RMS } \\
\text { (nm) }\end{array}$ & $\begin{array}{ll}20.8 & \pm \\
4.3 & \end{array}$ & $\begin{array}{ll}19.1 & \pm \\
1.3 & \end{array}$ & $\begin{array}{ll}35.7 & \pm \\
7.7 & \end{array}$ & $\begin{array}{ll}37.0 & \pm \\
4.6 & \end{array}$ & $6.4 \pm 4.3$ & $7.1 \pm 3.8$ & $6.2 \pm-3.0$ & $6.7 \pm 2.8$ \\
\hline $\begin{array}{l}\text { ADev } \\
(\mathrm{nm})\end{array}$ & $\begin{array}{ll}15.3 & \pm \\
3.8 & \end{array}$ & $\begin{array}{ll}15.1 & \pm \\
1.3 & \end{array}$ & $\begin{array}{ll}22.3 & \pm \\
5.7 & \end{array}$ & $\begin{array}{ll}28.1 & \pm \\
6.6 & \end{array}$ & $3.8 \pm 3.4$ & $4.7 \pm 3.0$ & $3.3 \pm 1.4$ & $5.2 \pm 2.2$ \\
\hline
\end{tabular}




\section{University Library}

\section{- M I I E E R VA \\ A gateway to Melbourne's research publications}

Minerva Access is the Institutional Repository of The University of Melbourne

Author/s:

Boni, LJ;Sanchez-Ferrer, A;Widmer, M;Biviano, MD;Mezzenga, R;Windhab, EJ;Dagastine,

RR;Fischer, $P$

Title:

Structure and Nanomechanics of Dry and Hydrated Intermediate Filament Films and Fibers Produced from Hagfish Slime Fibers

Date:

2018-11-28

Citation:

Boni, L. J., Sanchez-Ferrer, A., Widmer, M., Biviano, M. D., Mezzenga, R., Windhab, E. J., Dagastine, R. R. \& Fischer, P. (2018). Structure and Nanomechanics of Dry and Hydrated Intermediate Filament Films and Fibers Produced from Hagfish Slime Fibers. ACS APPLIED MATERIALS \& INTERFACES, 10 (47), pp.40460-40473. https://doi.org/10.1021/ acsami.8b 17166.

Persistent Link:

http://hdl.handle.net/11343/243033 